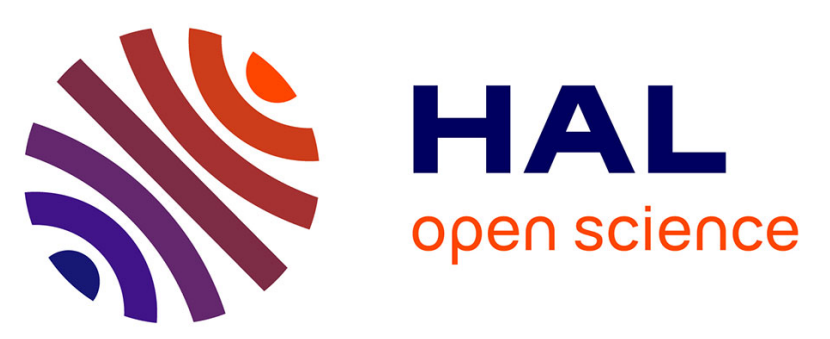

\title{
Coherent and turbulent processes in the bistable regime around a tandem of cylinders including reattached flow dynamics by means of high-speed PIV
}

M. Elhimer, Gilles Harran, Yannick Hoarau, Sébastien Cazin, Moïse Marchal, Marianna Braza

\section{To cite this version:}

M. Elhimer, Gilles Harran, Yannick Hoarau, Sébastien Cazin, Moïse Marchal, et al.. Coherent and turbulent processes in the bistable regime around a tandem of cylinders including reattached flow dynamics by means of high-speed PIV. Journal of Fluids and Structures, 2016, 60, pp.62-79. 10.1016/j.jfluidstructs.2015.10.008 . hal-02124846

\section{HAL Id: hal-02124846 \\ https://hal.science/hal-02124846}

Submitted on 9 May 2019

HAL is a multi-disciplinary open access archive for the deposit and dissemination of scientific research documents, whether they are published or not. The documents may come from teaching and research institutions in France or abroad, or from public or private research centers.
L'archive ouverte pluridisciplinaire HAL, est destinée au dépôt et à la diffusion de documents scientifiques de niveau recherche, publiés ou non, émanant des établissements d'enseignement et de recherche français ou étrangers, des laboratoires publics ou privés. 



\title{
Coherent and turbulent processes in the bistable regime around a tandem of cylinders including reattached flow dynamics by means of high-speed PIV
}

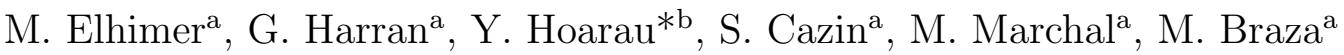 \\ ${ }^{a}$ Institut de Mécanique des Fluides de Toulouse, IMFT-UMR CNRS 5502, France \\ ${ }^{b} I C U B E$, Laboratoire des sciences de l'ingénieur, de l'informatique et de l'imagerie, \\ Université de Strasbourg, UMR CNRS 735\%, France
}

\begin{abstract}
The turbulent flow around two cylinders in tandem at the sub-critical Reynolds number range of order of $10^{5}$ and pitch to diameter ratio of 3.7 is investigated by using time-resolved Particle Image Velocimetry (TRPIV) of $1 \mathrm{kHz}$ and $8 \mathrm{kHz}$. The bi-stable flow regimes including a flow pattern I with a strong vortex shedding past the upstream and the downstream cylinder, as well as a flow pattern II corresponding to a weak alternating vortex shedding with reattachment past the upstream cylinder are investigated. The structure of this "reattachment regime" has been analyzed in association with the vortex dynamics past the downstream cylinder, by means of POD and phase-average decomposition. These elements allowed interconnection among all the measured PIV planes and hence analysis of the reattachment structure and the flow dynamics past both cylinders. The results highlight fundamental differences of the flow structure and dynamics around each cylinder and provide the 'gap' flow nature between the cylinders. Thanks to a high-speed camera of $8 \mathrm{kHz}$, the shear-layer vortices tracking has been possible downstream of the separation point and the quantification of their shedding frequency at the present high Reynolds number range has been achieved. This issue is important regarding fluid instabilities involved in the fluid-structure interaction of cylinder arrays in nuclear reactor systems, as well as acoustic noise generated from the tandem cylinders of a landing gear in aeronautics.
\end{abstract}

\footnotetext{
${ }^{*}$ Corresponding author.

E-mail address: hoarau@unistra.fr.
} 
Keywords: Tandem cylinder, Time resolved PIV, phase-average decomposition, POD

\section{Introduction}

Multiple cylinder structures can be found in the heat exchanger designs for the cooling systems of vapor nuclear reactors, offshore structures and cables, in both air and water flow. In many of these engineering applications, the instability modes developed in the fluid part involve von Kármán vortex shedding as well as shear-layer instabilities and their interaction among them and with the random turbulence. These instabilities are responsible for structural coupling leading to amplification of fluid-structure interaction instabilities, which may lead to flutter and damage of the solid structures. These problems are related with Vortex-Induced Vibration (VIV), Movement Induced Vibration (MIV) and noise. Examples of these fluid-elastic instability in heat exchanger tube arrays subjected to a cross-flow are studied by Granger and Paidoussis (1996), Paidoussis and Price (1988), Connors (1970). More recently, an experimental study of the flow instabilities in the first rows of tube banks is carried out by Olinto et al (2009) by using hot-wire velocimetry in an aerodynamic channel and flow visualizations in a water channel. In the wind channel, three tube banks with square arrangement and pitch to diameter ratios $\mathrm{P} / \mathrm{D}=1.26,1.4$ and 1.6 were studied in a Reynolds number range of $\left(7-810^{4}\right)$. Visualizations in a water channel complemented the analysis of the hot-wire results. A detailed time-frequency analysis was carried out. It was reported that the developed instabilities, generated after the second row of the tube bank, propagated to the interior of the bank. Secondary flow instabilities are also depicted due to three-dimensional effects. Experimental studies by Mahon et al (2012) of the surface pressure on a cylinder in a rotated triangular tube array $(\mathrm{P} / \mathrm{D}=1.375)$ with air cross-flow depicted a jet switching which results in large asymmetry in the pressure distribution even in a geometrically symmetric configuration and yields large lift force fluctuations which play an important role for design aspects against failure.

Numerical studies by Khalifa et al (2013) developed a computational fluid dynamics (CFD) model, experimentally validated to investigate the fluidelastic instability appearing as a critical flow induced vibration mechanism in tube and shell heat exchangers. The interaction between tube vibrations and 
flow perturbations in the low reduced velocities range of (1-6) and Reynolds numbers $(2000-12000)$ has been investigated. This study analyses in particular the physical nature of the time-lag between tube vibration and fluid response for a pitch ratio of 1.54 . This fluid-structure interaction instability as well as the phase-lag for reduced velocities in the range (2-10) have been also predicted by Shinde et al (2014) in 3D cylinder arrays, at an inter-tube Reynolds number of 60000 .

The evaluation of the critical parameters beyond which these fluid-structure instabilities amplify in the heat exchangers (reduced velocity and Scruton number) and lead to negative damping, motivated a number of experimental studies. The PIV studies and visualizations by Paul et al. (2007), Ikawi et al. (2004) at Reynolds number 5400 - 29700 shows a complex interaction between the shear layers and the von Kármán vortex streets in the flow field between the cylinders.

The tandem configuration is among the simplest forms of multiple cylinders structure, also including the side-by side configuration (Sumner et al (1999)). The two cylinders are in close proximity, arranged at arbitrary center-to-center spacing and zero angle of incidence relatively to the oncoming flow. This arrangement, in addition to the previously mentioned applications, corresponds to the two cylindrical supports of the aircraft's landing gear. This configuration at high Reynolds number is of crucial interest for the aerodynamic noise reduction produced by the downstream cylinder shear-layer instability, due to the interaction with the upstream cylinder. This configuration has been the object of detailed experiments in the NASALangley Research Center by using statistical (i.e. not time-resolved) PIV and wall pressure measurements by Jenkins et al. (2005), Lockard et al. (2007), Neuhart et al. (2009) as a canonical problem to identify and assess the effect of the interaction of unsteady flow among components of the landing gear on the airframe noise generation on large aircraft during approach. In particular, the results of these studies at Reynolds number 166000 with tripped boundary layer upstream of the separation, have been used as a test-case of the European research program Advanced Turbulence Simulations for Aerodynamic Application Challenges - ATAAC, allowing for validation of hybrid RANS-LES methods in aeronautics. A synthesis of the simulations results carried out for this problem can be found in Fu et al. (2012). In these studies it was shown that the interaction between the cylinders lead to complex vortex dynamics past the downstream cylinder and produce a well-distinct predominant frequency peak in the energy spectrum along the separated 
shear layer of the downstream cylinder. Furthermore, this configuration can be subjected to development of vibratory instability when the downstream cylinder is subjected to a single degree of freedom motion. The physical analysis of this instability and the critical assessment of flutter conditions are also of high interest in the context of the landing gear design. Numerical simulations of this tandem configuration with 1 DOF have been carried out by Ferreira-Perez (2013).

From the experimental point of view, the tandem arrangement has shown a large variety of flow patterns depending on the pitch (e.g. the distance between the cylinders) to diameter ratio, the Reynolds number and the upstream turbulence intensity. These patterns have been investigated by a number of studies (Igarashi (1981), Sumner et al (2000), Lin et al. (2002), Xu and Zhou (2004), Alam et al (2003), Alam and Meyer (2011)) who shows the existence of a Reynolds number dependent critical pitch for the transition from one pattern to another. The studies past the tandem cylinders show a complex interaction between the shear layer vortices and the Kármán vortex street in the flow field between the cylinders. Experimental studies by Lin et al. (2002) using a high-image-density PIV investigated the instantaneous and averaged velocity, vorticity and Reynolds stress flow patterns in the tandem cylinder configuration at a Reynolds number of 1000. Mahir and Rockwell (1996) had investigated the controlled excitation over a range of excitation frequency and phase-angle at Reynolds number of 160 .

A comprehensive review can be found in Sumner (2010) who suggested the following classification of the flow patterns around the tandem, depending on the pitch ratio in the high Reynolds number range. For the smallest values of the pitch ratio $L / D$ in the interval (1-2), the tandem acts as a single extended body because the downstream cylinder is located in the vortex formation zone of the upstream cylinder. This flow regime is called extended body regime. The separated shear layers from the upstream cylinder wrap around the downstream cylinder without any reattachment onto its surface, before rolling up alternately into Kármán vortices past the downstream cylinder. The flow between the two cylinders is almost stagnant but may behave as a cavity flow. For medium pitch ratio, $L / D$ in the interval of (2-5), the shear layers from the upstream cylinder reattach onto the downstream cylinder, with the formation and shedding of eddies in the gap region between the two cylinders. A wide variety of flow regimes can be observed in this case, depending on the experimental parameters and leading to different flow patterns including a bistable regime as detailed in the next paragraph. A first 
classification of these regimes was made by Igarashi (1981), who characterized the existence of two vortex shedding regimes past each cylinder as the co-shedding regime and an intermittent transition towards a bistable regime in random occurrence, where the vortex shedding past the upstream cylinder is attenuated. The reattachment position and the flow structure of this regime can vary substantially in terms of fluctuation levels and symmetry, depending on the Reynolds number. In this context, the variety of the gap flow structure (e.g. between the cylinders) has been confirmed from flow visualization by Lin et al. (2002). Finally, at higher pitch ratios, $L / D>5$, the downstream cylinder is sufficiently far from the upstream one; therefore the alternating vortex shedding can be fully developed past the upstream cylinder as well as past the downstream cylinder. This cylinder is located outside the vortex formation region of the upstream cylinder in this case and is subjected to the periodic impingement of shed vortices from the upstream cylinder.

The transition to and from the co-shedding regime is known to be bistable, with intermittent appearance of the two flow patterns (Igarashi (1981), Xu and Zhou (2004)). This bistable regime appears in several experiments with different blockage and aspects ratios (Sumner (2010)). In this bistable regime, the flow around the tandem switches randomly between reattachment and co-shedding patterns. Steady, alternate and intermittent reattachment of the shear layers was observed according to different Reynolds numbers, yielding different separation dynamics past the upstream cylinder. For all the pitch ratios in the present interval it was found that the von Kármán vortex shedding was clearly developed past the downstream cylinder (Sumner (2010)). The alternating reattachment behavior is observed when the shear layers alternately reattach in synchronization with Kármán vortex shedding from the downstream cylinder. This behavior was observed experimentally for pitch ratios $1.1<L / D<1.6$ by Igarashi (1981) as well as for pitch ratios $L / D<2$ and at Reynolds number $R e=6.5 \cdot 10^{4}$ by Alam et al (2003). Kitagawa and Ohta (2008) observed the same behavior from LES numerical simulations at lower Reynolds numbers $\left(R e=2.2 \cdot 10^{4}\right)$ for $L / D=2$. When the reattachment alternates, all these studies showed the appearance of alternating vortices in the gap between the two cylinders.

Interestingly, the bistable regime had been also observed for tandem plates and tandem prisms (Kim et al. (2008), Auteri et al (2008)) in critical Reynolds number conditions. When two cylinders in tandem are sufficiently far apart, the shear layers separating from the upstream cylinder reattach 
onto the downstream one. This reattachment regime has been investigated by several authors but its structure remains elusive. Moreover, the studies by Khalifa et al (2013); Mahon et al (2012); Olinto et al (2006, 2009); Keogh et al. (2014) indicate the appearance of bistable regimes in cylinder array configurations. Therefore, the bi-stable regime which will be analyzed in the present article concerning the tandem cylinders at a higher pitch ratio is potentially relevant to heat exchanger tube arrays.

At large pitch ratios, the flow between the two cylinder ceases to be periodic and becomes quasi-symmetric and quasi-steady. Thus the different authors qualify the reattachment as steady and observe that the two shear layers seem reattached almost simultaneously onto the downstream cylinder. Indeed Igarashi (1981) and Alam et al (2003) observe the appearance of quasi-steady eddies near the upstream face of the downstream cylinder for intermediate pitch ratios between $1.6<L / D<2.3-2.4$ in Igarashi (1981) and up to $L / D=2.5$ in Alam et al (2003). These vortices are however not observed in the LES simulations of Kitagawa and Ohta (2008) for the pitch $L / D=2$, although these authors agree on the steadiness of the reattachment. In Kitagawa's results, although small vortices where found intermittently around the front of the downstream cylinder due to the shear layer reattachment, these vortices did not develop but disappeared. For these pitch ratios, Zhou and Yiu (2006) noted that the reattachment occurs more often on the downstream side of the cylinder; the shear layers interfere with the boundary layer development and separation thus weakening the strength of the Kármán vortices formed behind this cylinder. In contrast, for larger pitch ratios $L / D=3-5$ these authors show that the influence on the downstream cylinder's boundary layer development is weaker since the reattachment occurs more often on the downstream cylinder's upstream side; thus the Kármán vortices are strengthened. The importance of the reattachment position on the flow around the downstream cylinder have also been stressed by Alam et al (2003), who highlights a strong relationship between the pitch ratio, the separation point and the fluctuating lift and drag coefficients. This author concluded that the fluctuating forces acting on the downstream cylinder depend on the reattachment position and they increase when this position precedes forward and vice versa. As the pitch increases, the gap flow becomes progressively more fluctuating. Kitagawa and Ohta (2008)) show that at $L / D=2.5$, the flow is almost the same as in the $L / D=2$ case, but the reattached shear layer becomes unstable and fluctuates. For larger pitch values, $L / D \approx 3$, Igarashi (1981), Kitagawa and 
Ohta (2008) note the intermittent appearance of a second flow pattern consisting of vortex shedding from the upstream cylinder. These vortices are convected towards the downstream cylinder.

As the previous review shows, the flow structure and dynamics in the gap region are still ambiguous and indeterminate, especially at larger pitch ratios within the reattachment regime. This is mainly due to the scarcity of data from quantitative imaging techniques such as high speed PIV, which may enable the resolution of both the spatial structure and temporal evolution of the gap flow. The aim of our ongoing experimental works, part of which is presented in this article, is to provide such data. In addition, the tandem configuration provides an excellent test for turbulence modeling validation. Thus, these PIV measurements may also provide a large database that can be used for comparison with numerical simulations, as in the study by Perrin et al. (2007) for a single cylinder. A considerable part of the present study have been carried out with a pitch ratio in the critical range in respect of the bistable regime. The two observed flow patterns will then be discussed and a special attention will be paid for the reattachment pattern. The purpose of the present study is to clarify this structure, by means of high-speed particle image velocimetry (PIV) and data analysis based on Proper Orthogonal Decomposition and phase-averaging. The tandem's pitch to diameter ratio is taken $L / D=3.7$ and the Reynolds number close to $\approx 10^{5}$ corresponding to sub-critical range. This article aims at highlighting fundamental differences of the flow structure and dynamics around each of the two cylinders and providing a detailed description of the gap flow nature and its phase relation with the wake of the downstream cylinder. Moreover, thanks to a high data acquisition camera, this paper aims at capturing the dynamics of the separated shear layer past the upstream cylinder. Using both, instantaneous and phase-averaged velocity and vorticity fields, the development of the shear layers from the separation to the reattachment will be studied, as well as the structure of the gap vortices and their coupling mechanism with the reattachment and the vortex shedding.

The present article is organized as follows: the second section is dedicated to the presentation of the high speed PIV set-up and the experimental conditions, as well as the detailed description of the the post-processing techniques, namely the POD (Proper Orthogonal Decomposition) and the phase-averaging. In the third section, the results of the present experimental investigations are discussed, followed by the conclusions. 


\section{Experimental set-up, conditions and techniques}

\subsection{Description of the experiments: experimental and PIV setups}

The experiments were undertaken in the wind tunnel S4 of IMFT, which delivers an upstream velocity with low turbulent fluctuations (the turbulence intensity has been measured by means hot wire anemometer and found to be of order 0.15 percent of the upstream velocity). The test section is of $58 \mathrm{~cm}$ width and $72 \mathrm{~cm}$ height. Two transparent Plexiglas cylinders of diameter $8 \mathrm{~cm}$ have been used, yielding an aspect ratio of 7.25 and a blockage ratio of 0.11 . This compares well with Lin et al. (2002) experiments were the aspect ratio is 8.3 and the blockage ratio 0.1 .

In the study by Igarashi (1981), the aspect and blockage ratios were 4 and 0.06 respectively. In the study by Xu and Zhou (2004), these values were 40 and 0.025 respectively. Thus the blockage ratio in the present study is comparable to other experiments in the literature.

\begin{tabular}{|l|l|l|l|l|l|l|}
\hline Plan & Dimensions & Used camera & Sensor resolution & Frequency & $\begin{array}{l}\text { Duration of } \\
\text { one acquisition }\end{array}$ & $\begin{array}{l}\text { Number of } \\
\text { acquisitions }\end{array}$ \\
\hline 1 & $0.88 D \times 0.55 D$ & PHANTOM V1610 & $1280 \times 800$ pixels & $8000 \mathrm{~Hz}$ & $8.3 \mathrm{~s}$ & 1 \\
\hline 2 & $2 D \times 2 D$ & PHOTRON RS3000 & $1024 \times 1024$ pixels & $1000 \mathrm{~Hz}$ & $3 \mathrm{~s}$ & 10 \\
\hline 3 & $2 D \times 2 D$ & PCO DIMAX & $2048 \times 2048$ pixels & $1000 \mathrm{~Hz}$ & $6.3 \mathrm{~s}$ & 5 \\
\hline 4 & $1.8 D \times 1.8 D$ & PHOTRON RS3000 & $1024 \times 1024$ pixels & $1000 \mathrm{~Hz}$ & $3 \mathrm{~s}$ & 10 \\
\hline 5 & $2 D \times 2 D$ & PHOTRON RS3000 & $1024 \times 1024$ pixels & $1000 \mathrm{~Hz}$ & $3 \mathrm{~s}$ & 10 \\
\hline
\end{tabular}

Table 1: PIV campaigns

In the present study, the pitch to diameter ratio of the tandem is $L / D=$ 3.7 corresponding to a gap distance of $21.6 \mathrm{~cm}$ wide. The cylinders are positioned in cross-flow at the mid-height of the test section and spanned over its width. The upstream velocity in the wind tunnel was measured using a Pitot tube with pressure plugs at the inlet and the outlet of the contraction section and was controlled by changing the fan angular velocity. Time-resolved PIV measurements have been carried out at four different upstream velocities $U=5.99 \mathrm{~m} / \mathrm{s}, 9.03 \mathrm{~m} / \mathrm{s}, 20.6 \mathrm{~m} / \mathrm{s}$, and $25.5 \mathrm{~m} / \mathrm{s}$ corresponding to the Reynolds numbers $R e=31200, R e=47000, R e=107300$ and $R e=132800$ respectively, based on the cylinders diameter. For all these Reynolds numbers, the flow is sub-critical and falls into the "TrSL regime" where the transition occurs in the separated shear layer (Zdravkovich (1991)). In the present article, the results obtained with the upstream velocity $U=20.6 \mathrm{~m} / \mathrm{s}$ and the Reynolds number $R e=107291$ are reported.

The velocity field measurements have been realized by using a high speed PIV set-up that includes a double frame high-speed camera, a two cavity 
Laser Quantronix Darwin and a synchronizer from LAVISION. Using a combination of spherical and cylindrical lenses, the Laser beam was expanded into a light sheet of $2 \mathrm{~mm}$ thickness. The energy of the Laser beam delivered by each cavity was set at $25 \mathrm{~mJ}$. The flow was seeded with water-alcohol droplets by using a fog generator. The droplet size distribution was measured using a Malvern granulometer and were of order of $3.4 \mu \mathrm{m}$ in average with a small standard deviation.

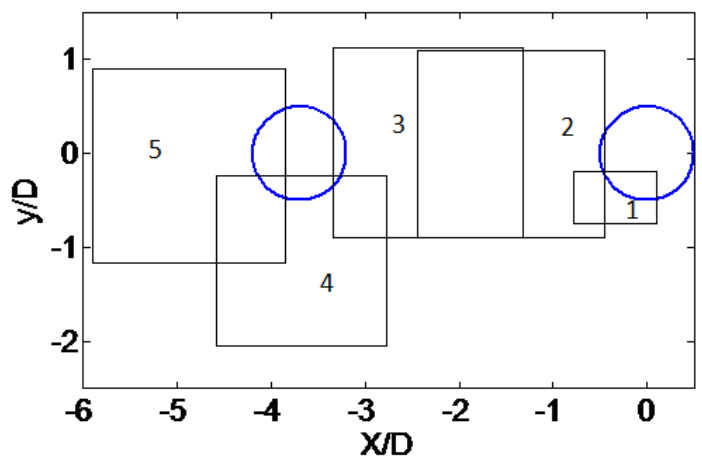

Figure 1: Visualization planes

Five PIV campaigns have been carried out, corresponding to five different measurement planes (Figure 1) and using three different high-speed cameras, as shown in Table 2.1. The measurement areas are the wake and the separation region of the upstream cylinder (areas 1 and 2), as well as the wake and the lower reattachment region of the downstream cylinder (areas 34 and 5 respectively). The upper reattachment region was not illuminated by the Laser sheet due to its blockage by the cylinder.

The acquisition frequency was set to $2000 \mathrm{~Hz}$ for the RS300 and the DIMAX cameras and to $16000 \mathrm{~Hz}$ for the V1610 camera, thus the frequency of the PIV acquisitions was $1000 \mathrm{~Hz}$ and $8000 \mathrm{~Hz}$ respectively. The high frequency of the PIV measurements allowed extracting the time-dependent signals at any location of the measurement planes. For each location the time series from the ten acquisitions were concatenated in order to form a sufficiently long time series. The PSD of these time series was computed 
using spectral averaging on multiple windows (Marple (1987)) with nonoverlapping Hamming windows of a size corresponding to 1024 samples.

The image processing and the cross-correlation analysis were realized by using the DAVIS 8 software of La Vision. A slight inhomogeneity gradient of the Laser's light appears in the measuring plane, which is due to an intrinsic characteristic of the Laser source related to the nominal distribution of the energy in the Laser's beam. Furthermore, one of the cameras used, the RS3000 type, presents an intrinsic slight inhomogeneous intensity response yielding a slight absorption of the signal's intensity in the borders of the measuring plane. These effects create slight fringes of more lighted zones and darker ones but this pattern is quite weak and has no significant impact on the measurements quality, whose "success rate" was higher than $93 \%$ before "false" vectors are removed. These slight inhomogeneities due to the background's grey level of each raw image have been removed by considering an order of twenty consecutive images in the vicinity of the treated image. The minimum grey level from which passes each pixel on these images has been recorded and therefore an information on the energy distribution in the image's background under treatment has been obtained. By subtracting this to each treated image, these lighting effects have been removed and they did not influence in this way the PIV correlations. An iterative cross-correlation analysis with decreasing interrogation windows was used to compute the displacement vectors from the processed images and a final interrogation window size of $12 x 12$ pixels with an overlap of $50 \%$ and $75 \%$ was used. Finally the resulting vector fields were post-processed using an iterative median filter in order to detect and replace "false" vectors (Adrian and Westerweel (2010)) The percentage order of vectors replaced by interpolation was less than $6 \%$.

\subsection{PIV results post-processing: phase averaging and Proper Orthogonal De- composition (POD) analysis}

\subsubsection{Phase-averaging of quasi-periodic turbulent flows}

The turbulent flow in the wake of bluff bodies consists of a regular largevortex shedding process. As a consequence, the flow contains a dominant quasi-periodic component, superimposed upon random fluctuations. The quasi-periodic motion is characterized by a well defined frequency peak, while the random frequencies spread over a large continuous spectrum. An appropriate operator to extract this periodic feature of the flow is the phaseaveraging, which consists of decomposing each of each instantaneous velocity 
field into a periodic part and a fluctuating one. This operator, originally introduced by Hussain and Reynolds (1970) in turbulent shear flows, has been extensively used by different investigators for the cylinders wake study as for example (Cantwell and Coles (1983), Kourta et al. (1987), Van Oudheusden et al. (2007), Perrin et al. (2007)).

Using a phase indicator, each instantaneous velocity field at time $t$ is matched with a phase $\phi(t)$ of the vortex shedding cycle. The phase averaged field for each phase angle $\phi$ is then the average over all the instantaneous velocity fields belonging to this phase. Thus, as for the classical Reynolds decomposition, any instantaneous velocity field can be written as the sum:

$$
u(x, t)=\tilde{u}(x, \phi(t))+u^{\prime \prime}(x, t)
$$

where $\tilde{u}$ is the phase-averaged velocity field at the phase of the cycle $\phi(t)$ corresponding to the time $t$, while the residual part $u$ " is the fluctuation about the phase. It should be noted that the phase-average operator is strictly periodic, whereas in the physical reality, there exist irregularities in the vortex shedding period, known as the "phase-jitter", Perrin et al. (2007) among other. This is due to the organized part coupling with turbulent fluctuations and chaotic processes, particularly appearing in high Reynolds number flows. This phase-jitter, as well as higher order harmonics and the random motion are all included in the residual part $u^{\prime \prime}$ of the velocity fluctuation.

The phase averaging procedure requires a "pilot signal" from which the phase of the vortex shedding cycle can be identified at any time. This pilot signal can either be a locally probed quantity (velocity, pressure ...) or a global indicator extracted from each velocity field. For an ensemble of instantaneous flow field snapshots provided by a time resolved PIV, the latter is a more advantageous method adopted in the present case, because it does not need a prior knowledge of the flow field for selecting a suitable probing region. In the present study, a global phase identification method has been used, in which the phase of each snapshot is determined from its projection on the first two POD modes of the snapshot series, according to Van Oudheusden et al. (2007). These two modes are considered to represent the basic contribution to the quasi-periodic component of the motion; the details of the procedure will be described in the third section of this article. By means of these two modes and the corresponding temporal POD coefficients, a phase angle corresponding to each snapshot can be defined, which allows therefore can therefore phase-classification of each snapshot. The main advantage of 
this global indicator is that the POD filters out the velocity fluctuations that are incoherent with the large scale motion by representing them with higherorder modes. Furthermore, results from Perrin et al. (2007) show that the effects of the phase jitter are alleviated by using this method.

\subsubsection{Proper orthogonal decomposition (POD)}

The Proper Orthogonal Decomposition is a procedure for extracting a basis for a modal decomposition from an ensemble of fields, according to Berkooz et al (1993). In the context of fluid dynamics and PIV measurements, its attractiveness lays on the fact that it provides a decomposition of the fluctuating part of the velocity field by means of the most energetic modes, since its first modes contain the dominant spatial and temporal flow features. In the case of a quasi-periodic flow, this means that the vortex shedding can be described by a reduced number of modes, thence providing a reduced order model of these flows. The POD thus provides both an identification of dominant coherent flow patterns and a basis for reduced order modelling, able to capture the most energetic modes governing the flow system.

The POD is carried out by solving an eigenvalue problem where the kernel is the two-point velocity correlation tensor. The eigenvectors of this problem are the spatial modes $\phi_{i}(x)$ and the corresponding eigenvalues $\lambda_{k}$ represent the energy contained by each mode. Given this basis, the temporal modes $a_{i}(t)$ are the projection of the instantaneous velocity field at time $t$ on the spatial modes $\phi_{i}(x)$. The velocity fields can be reconstructed with the desired accuracy by using an appropriate number of modes $N$ :

$$
u(x, t)=U(x)+u^{\prime}(x, t) \approx U(x)+\sum_{i=1}^{N} a_{i}(t) \phi_{i}(x)
$$

where $U(x)$ is the time-averaged velocity field. In the present study, the POD mode evaluation suggested by Sirovich (1987) has been used to solve

the eigenvalue problem. As will be shown further in this study, only the two first POD modes are required to accurately reconstruct the vortex shedding, as shown in the case of a single cylinder by Van Oudheusden et al. (2007) and Perrin et al. (2007). 


\section{Results and discussions}

This section aims at highlighting the existence of a bistable mode of the flow around the tandem of cylinder beyond of a critical Reynolds. Two flow patterns will be compared in presence of this bistable mode and it will be shown that the first corresponds to the "co-shedding" regime while the second corresponds to the "reattachment regime". This section is dedicated to investigate the dynamics of these regimes and particularly of the reattachment regime.

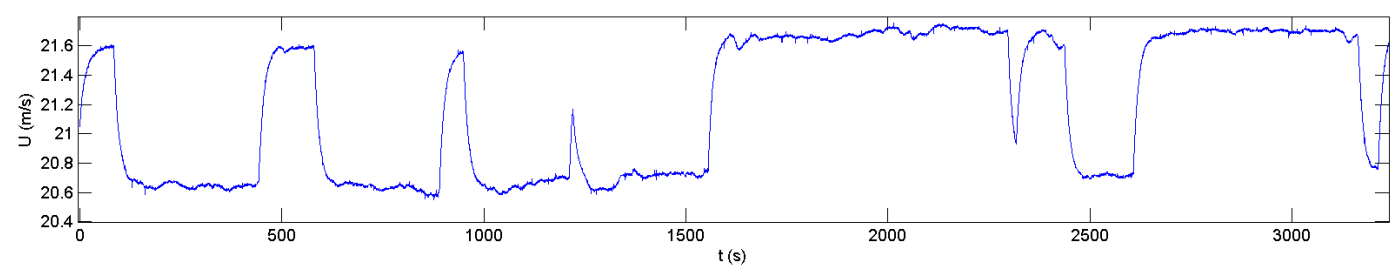

Figure 2: Time series of the upstream velocity in the wind tunnel in the bistable regime. The duration of the recording is 54 minutes and the sampling rate is of $5 \mathrm{~Hz}$. The velocity switches between two values $20.6 \mathrm{~m} / \mathrm{s}$ and $21.6 \mathrm{~m} / \mathrm{s}$ which correspond respectively to the flow pattern I and the flow pattern II

\subsection{Bistable flow around the tandem of cylinder}

\subsubsection{Description of a bistable regime}

A bistable flow regime was observed in the present experiments: This regime is characterized by the coexistence of two flow patterns which correspond to those observed before and after the critical pitch ratio, that is to say respectively the "co-shedding mode" and the "reattachment" mode (see the introduction); in the following, the first configuration will be also called "flow pattern I (FPI)" and the second "flow pattern II (FPII)". In the literature, it is shown that the average drag on the whole of the tandem in the first configuration is larger than in the second. Thus, around the critical pitch ratio, Alam et al (2003) measure an increase in the mean drag coefficient of approximately $50 \%$ for the upstream cylinder and of $140 \%$ for the downstream one during the transition from the reattachment mode to the co-shedding mode. In our experiments, this change of drag at the transition results in a variation of the pressure loss in the wind tunnel, which induces an upstream velocity modification at each transition between the two flow 
patterns. In order to illustrate this fact, the fan speed was fixed to reach an upstream velocity of $U=20.6 \mathrm{~m} / \mathrm{s}$, then the signal of the the upstream velocity measured by the Pitot tube was recorded during 54 minutes at a sampling rate of $5 \mathrm{~Hz}$. This signal is presented in Figure 2. During the recording, the temperature was stable $\left(19^{\circ} \mathrm{C}\right)$ and the fan operated in practically steady velocity, in respect of the certification measurements of the $\mathrm{S} 4$ wind tunnel of IMFT, which ensures a variability of the fan speed lower than $0.01 \%$

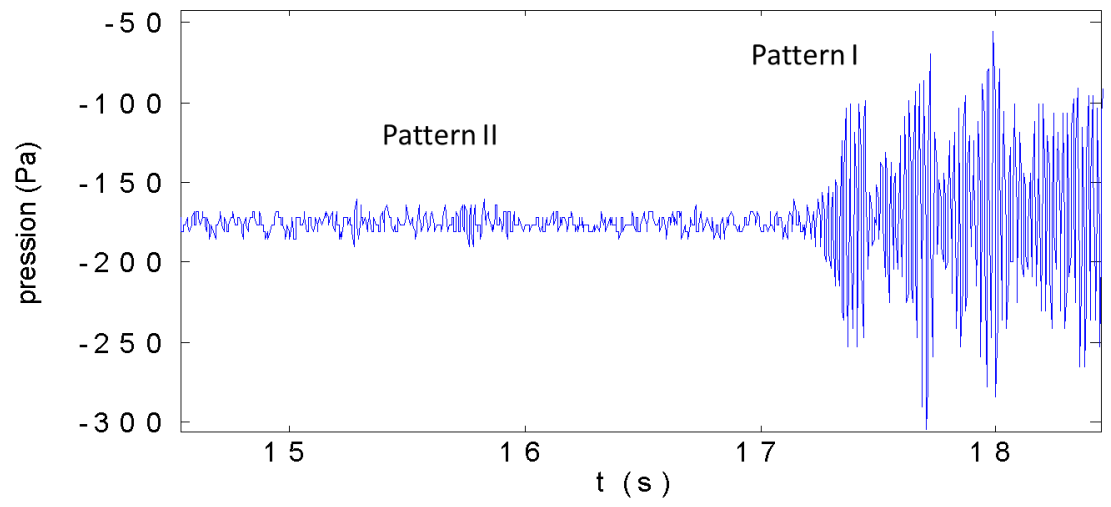

Figure 3: Surface pressure signal at the angular position of $\theta=28^{\circ}$ on the upstream cylinder showing the switch between the two flow patterns. Here the cylinder has a diameter of $D=10 \mathrm{~cm}$ and the Reynolds number is $R e=166000$. Due to the absence of high-amplitude coherent structures near the cylinder in the flow pattern II, the pressure fluctuations are weaker than in pattern I.

This paper aims at attributing a particular attention to the existence of two flow stages at $20.6 \mathrm{~m} / \mathrm{s}$ and $21.6 \mathrm{~m} / \mathrm{s}$ (with weak fluctuations around these values), between which the flow switches; the low stage corresponds to the flow pattern I (high pressure loss) while the high stage corresponds to the flow pattern II (low pressure loss). It is shown that the transition between the two patterns always induces a modification of the upstream velocity close to $1 \mathrm{~m} / \mathrm{s}$ thus showing the repeatability of this switching phenomenon. However, this phenomenon is clearly not periodical since the time between two transitions and the lifetime of each flow pattern are not repeatable. Indeed one observes nine transitions during the recording, each flow pattern having unequal duration ranging from less than $100 \mathrm{~s}$ to more than $500 \mathrm{~s}$. These transitions are fast because they are accomplished during about $40 \mathrm{~s}$. 
Moreover, "aborted transitions" occur when the flow approaches one of the two stages but does not reach it and returns towards the initial stage. This event occured three times in the present experiments, twice for the transition from FPI towards FPII and once for the transition from FPII towards FPI.

Another method of transition tracking is the fluctuating pressure signal recording on the upstream cylinder. This was undertaken in the present experiments for a cylinder's diameter of $10 \mathrm{~cm}$ and at a Reynolds number $R e=166000$. In Figure 3, a time series of the surface pressure at the angular position $\theta=28^{\circ}$ is shown. The switching is characterized by the fluctuating pressure amplitude change. Indeed the increase of pressure fluctuations is related to the appearance of the Von-Kármán mode near the upstream cylinder, which generates high periodic pressure fluctuations.
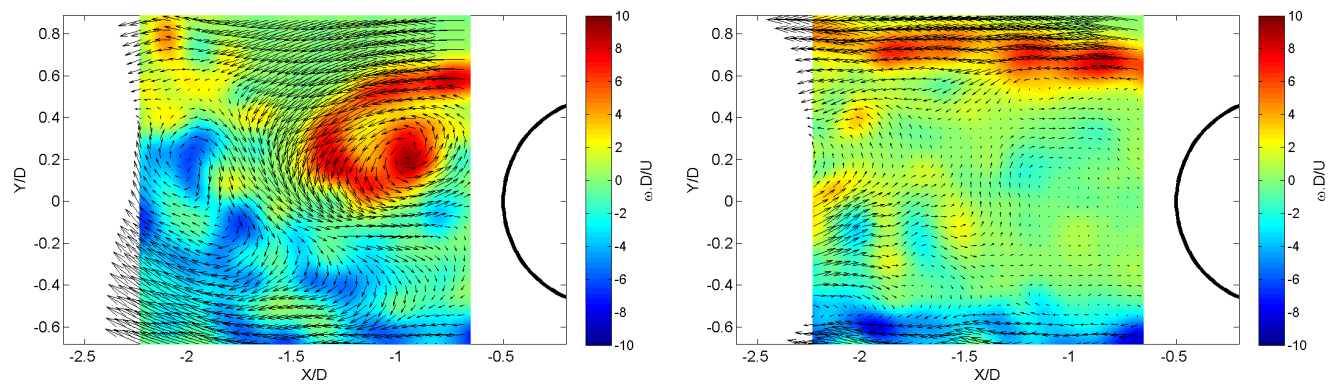

a)

b)

Figure 4: Instantaneous velocity (vectors) and vorticity (background) field past the upstream cylinder: a) flow pattern I; b) flow pattern II. The flow direction is from the right to left, as in the S4 wind tunnel of IMFT. In flow pattern I, large-scale alternating vortices are clearly visible, while the flow pattern II shows two horizontal shear layers, as well as weaker traces of large vortices. The fields were smoothed by Gaussian filtering for clarity

The bistable mode was observed only for the two higher Reynolds numbers, $R e=107291$ and $R e=132812$, which suggests the existence of a critical Reynolds number for the appearance of this mode. To detect the critical Reynolds number, the fan speed varied linearly corresponding to an upstream velocity variation from $9 \mathrm{~m} / \mathrm{s}$ to $22.6 \mathrm{~m} / \mathrm{s}$. During the experiment, the measured velocity should match this variation, however once the velocity reached $18 \mathrm{~m} / \mathrm{s}$, abrupt changes in the measured velocity were observed. The repeatability of this behavior has been verified. Hence, it can reasonably be deduced that this value is the critical velocity of the bistable mode appearance. 


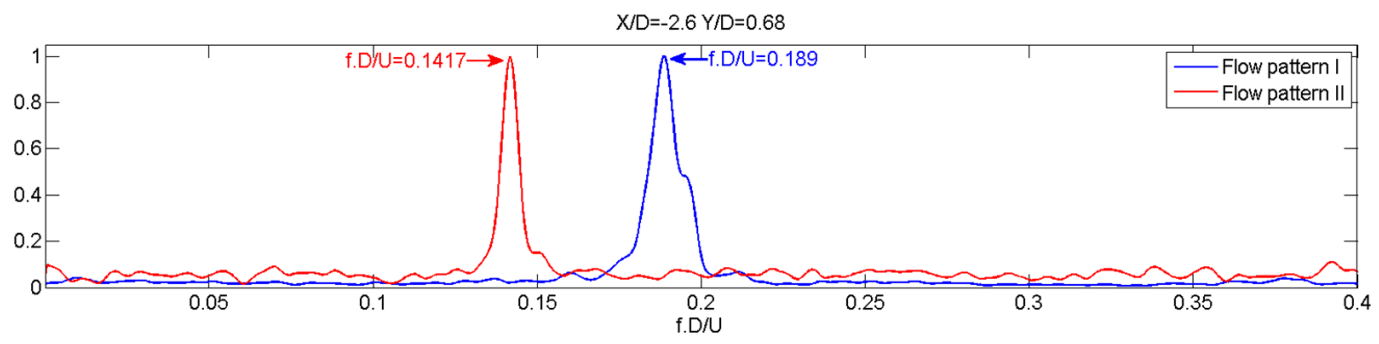

Figure 5: Spectra of the vertical velocity at position $X / D=-2.6, Y / D=0.68$, corresponding to plane 3 of figure 1 , for the two flow patterns of the bistable regime. The two configurations reveal a Strouhal number at two different frequencies.

\subsubsection{Comparison of the two flow patterns of the bistable regime}

The comparison of the instantaneous velocity fields in the wake of the upstream cylinder reveals the differences in the topology between the two patterns (Figure 4). For the flow pattern I, the shedding of large-scale vortices with a topology similar to the single cylinder's alternating vortices is observed. The instantaneous velocity fields for the flow pattern II shows two horizontal shear layers, which concentrate the vorticity and delimit a stagnation zone between them. These layers do not roll-up in the near wake and seem to keep fairly the same horizontal position. However traces of weaker alternating vortices can be distinguished and as the energy spectra show, there still exists a shedding frequency, which becomes more pronounced farther downstream. The signature of these vortices is visible indeed on the vertical velocity spectrum which presents a peak at the dimensionless frequency $f . U / D=0.141$. At the same position $X / D=-2.6, Y / D=0.68$, the spectrum in FPI shows a peak at a quite different frequency (Strouhal number) $f . U / D=0.189$ which is closer to the Strouhal number value for an isolated cylinder. These spectra confirm the existence of a quasi-periodic motion for the two configurations but having different topologies and dynamics. Figure 5 shows the spectra at the same spatial positions for both regimes. As will be detailed in the next section, there is an amplitude modulation of the von Kármán mode according to these regimes.

These differences are also visible in the time-averaged fields of the velocity components and Reynolds stresses presented in Figure 6, where the normalized horizontal mean velocity $U_{m} / U$, the normal stress $u^{\prime 2} / U^{2}$ and the shear stress $u v / U^{2}$ are shown for the two flow patterns in the wake of the upstream 

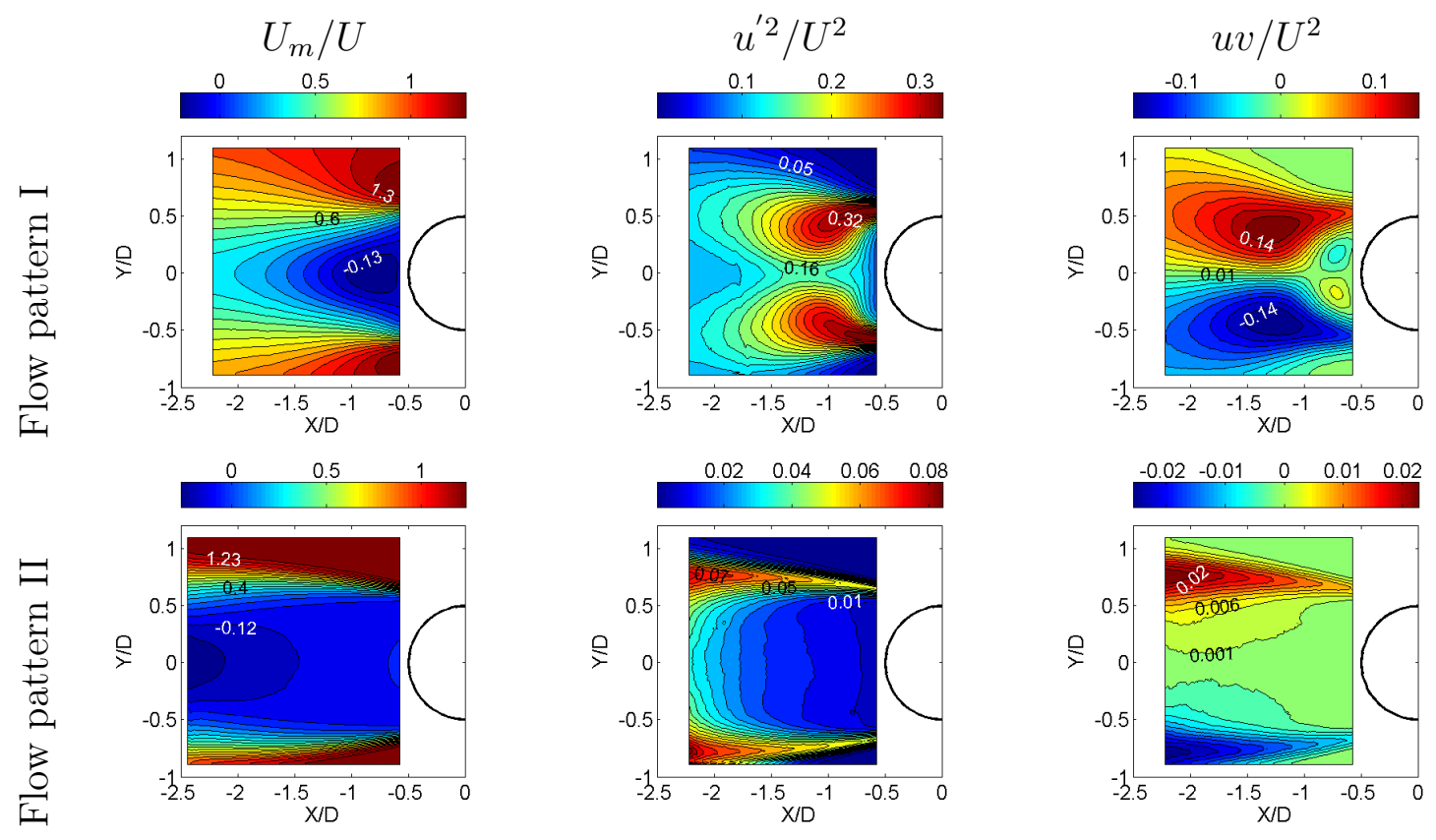

Figure 6: Time-averaged velocity fields in the wake of the upstream cylinder. Comparison between the two flow patterns; upper row : flow pattern I; lower row : flow pattern II. 
cylinder. For these results, it is confirmed that while the velocity statistics of FPI are qualitatively similar to the single cylinder case, the statistics of the second flow pattern show a distinct topology. In addition, the velocity fluctuation level is significantly lower in FPII in comparison with FPI, where the longitudinal and vertical Reynolds stresses are up to ten times larger than in FPII at the same location.

In FPI, the average velocity field $U_{m}$ is symmetric with respect to the axis $y / D=0$ and the irrotational region, the shear layers and the "stagnation zone" are clearly identified. In the irrotational region, the upstream flow is accelerated up to $1.3 U$ near the cylinder and beyond the position $y / D= \pm 0.8$, in agreement with the results of Braza et al. (2006) for an isolated cylinder at a close Reynolds number $R e=140000$. In addition, the recirculation length, defined as the distance between the axis of the cylinder and the point were $U_{m}=0$, is $l_{c}=1.27 \mathrm{D}$ which is in good agreement with the value $l_{c}=1.28 D$ obtained by Braza et al. (2006). Concerning FPII, two irrotational zones are identified at both sides of the upstream cylinder, were the mean longitudinal velocity reaches a higher value than in FPI: $1.4 U$ at $y / D= \pm 0.8$ on the line $s / D=0.71$. The shear layers located between $y / D= \pm 0.5$ and $y / D= \pm 0.8$ delimit a stagnation zone that spans over a larger area than in the first flow pattern. Farther away from the cylinder, the average velocity becomes negative; this suggests the existence of a downstream recirculation flow most probably generated by the reattachment of the shear layers on the downstream cylinder.

In both flow patterns, the normal stresses have a symmetrical distribution with respect to the axis $y / D=0$, while the shear stress has an anti-symmetric distribution. In FPII, the Reynolds stresses concentrate in the shear layers that thicken away from the cylinder due to the turbulent diffusion. Indeed, the shear layer thickness increases from $0.25 D$ at $x / D=-0.71$ to $0.5 D$ at $x / D=-1.5$. The Reynolds stresses are negligible close to the cylinder in the "stagnation zone" and increase as the recirculation flow gets closer. The Reynolds stresses in FPI are significantly larger compared to FPII, due to the presence of the large-scale vortices close to the upstream cylinder. In addition, FPI's Reynolds stress fields show a topology and normalized values close to the single cylinder' case.

Thus, while for the first flow pattern the wake of the upstream cylinder is qualitatively similar to the single cylinder case, the second flow pattern shows more intriguing features, which rise several questions about the reattachment structure. Therefore the focus is made on this flow pattern in the following 
sections of this article.

\subsection{Dynamics and topology of the reattachment pattern}

\subsubsection{POD analysis and phase averaging procedure}

The POD analysis was used here to calculate the phase-averaged flow fields past the two cylinders and in the gap area and to illustrate therefore the overall flow structure by "unifying" the different PIV fields, based on the present conditional operation. The POD results are shown first for the velocity fields in the wake of the downstream cylinder. Secondly, the evaluation of phase-averaging is carried out by means of the two first POD modes. Figure 7 shows the eigenmodes $\phi_{i}$ and temporal coefficients $a_{i}$ of the first POD modes $i=1,2,3,4,6,8,10,20$ computed from 3053 snapshots acquired at a sampling frequency of $1000 \mathrm{~Hz}$.

As expected, the first two POD modes show a significant periodicity and a topology consisting of large scale coherent vortices whose kernels are located on the $X$ axis, illustrating the existence of a large-scale vortex shedding. The two modes have close topologies and signal forms while being out of phase in time and space, as expected by the alternating character of the vortices in respect of the two-mode reconstruction. The first and second POD modes have largest contributions to the total kinetic energy. The temporal evolution of the higher-order modes is governed by more than one predominant frequency and there exist higher modes displaying a periodic character, as mode 6 for example. Moreover, theses modes seem to be associated with higher frequencies. These observations are confirmed by the spectra of the temporal coefficients of the first six POD modes (Figure 8). The spectra of the first two POD modes present a peak at the non-dimensional frequency $f . U / D=0.14$ while the modes 3,4 and 5 show peaks at the frequency $f . U / D=0.28$ of the first harmonic. For these modes, a co-existence of several frequency peaks can be noted. Finally the energy of each POD mode from 1 to 1000, normalized by the first mode $\lambda_{i} / \lambda_{1}$ is shown in Figure 9: The first two modes have close energies while the third mode is 10 times less energetic than the first; it can be noted that starting from this mode, the energy decreases as a function of the mode order, according to a power law with an exponent of 1.24 .

It can be seen that each of the two first POD modes have largest contributions to the total kinetic energy and contain the dynamics of the periodic movement as in the flow case around a single cylinder. Therefore, these two modes have been used for the phase identification of each snapshot. 

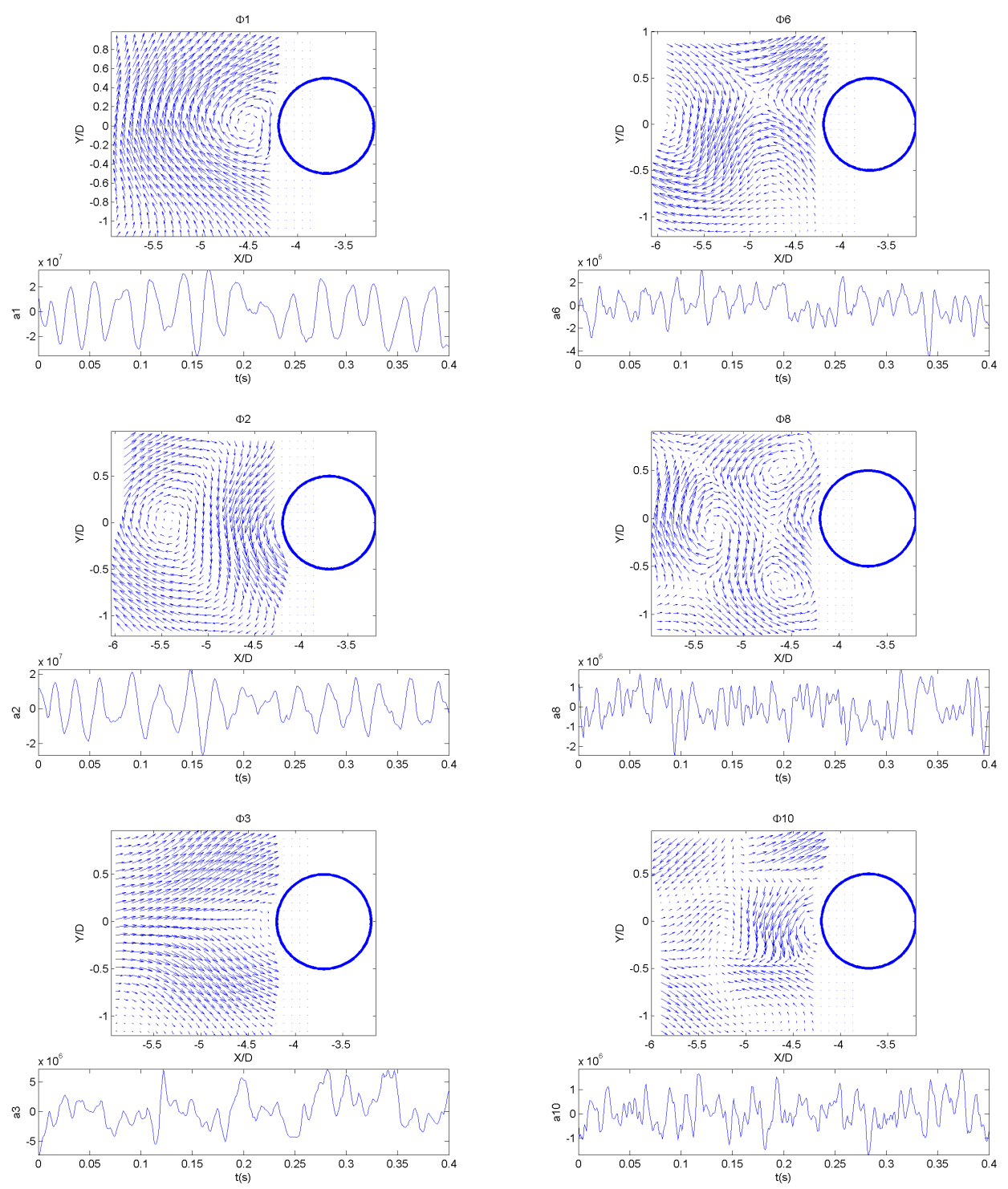

Figure 7: Eigenmodes $\phi_{i}$ and temporal coefficients $a_{i}$ of the first POD modes resulting from fluctuating velocity fields in the wake of the downstream cylinder in Flow pattern II (reattachment mode). 


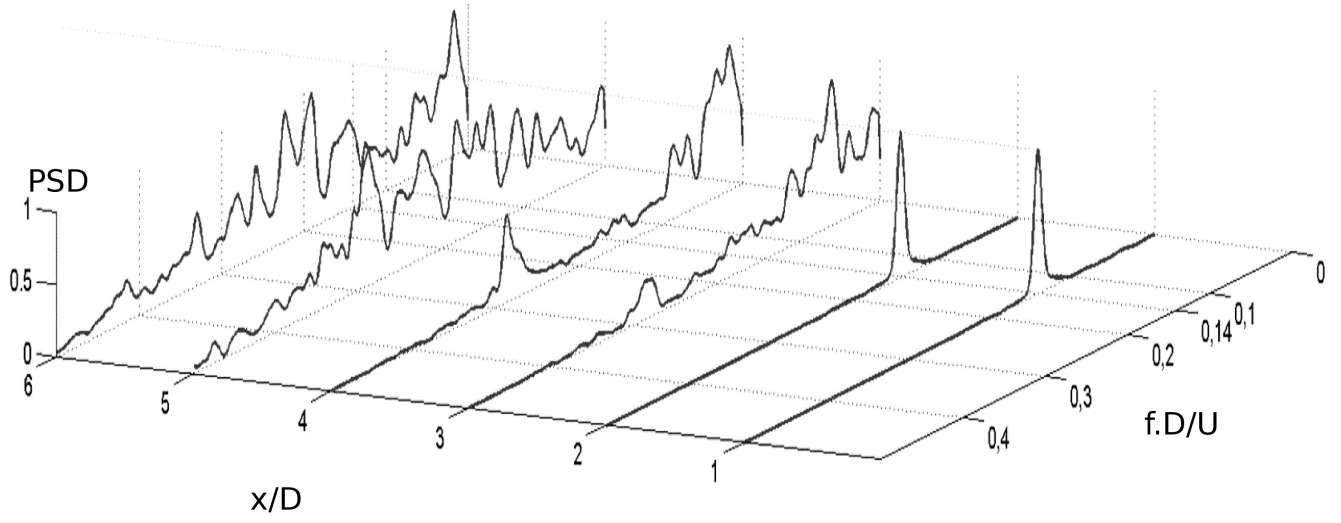

Figure 8: Spectra of the temporal coefficients of the six first POD modes. The Power Spectral Densities (PSDs) are normalised by their maximum values. 


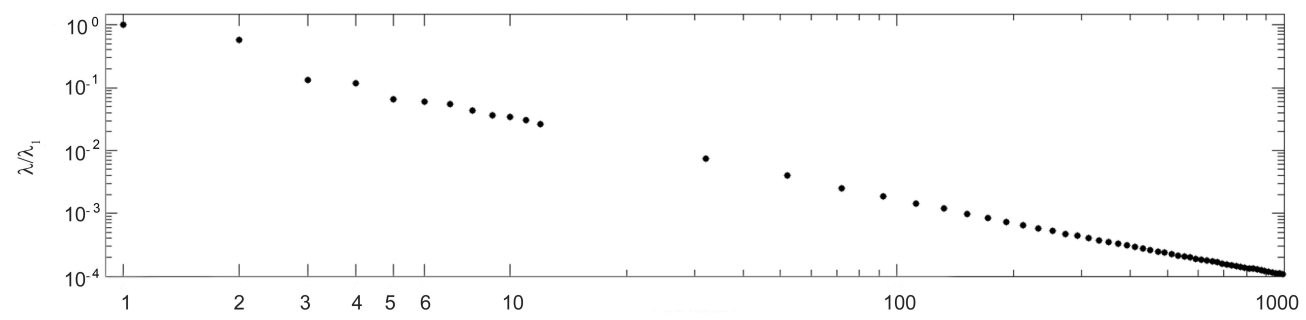

Figure 9: Energy distribution of the first 1000 modes POD, normalised by the first mode energy. The first two modes have close energy values. From mode 3, the energy decreases according to a power law with a coefficient of 1.24.

The temporal coefficients $a_{1}(t) a_{2}(t)$ are first considered for each snapshot by projecting it onto the first two POD modes computed from the 3053 snapshots of the first PIV data acquisition. The phase angle $\phi$ is extracted as being the angle in the normalized plane $\left(a_{1} / \sqrt{ }\left(2 \lambda_{1}\right), a_{2} / \sqrt{ }\left(2 \lambda_{2}\right)\right)$ hence $\phi=\arctan \left(a_{1} / \sqrt{ }\left(2 \lambda_{1}\right) / a_{2} * \sqrt{ }\left(2 \lambda_{2}\right)\right)$, according to Van Oudheusden et al. (2007); an example of the phase angle time series is shown in Figure 10a). A quasi-periodic cycle starts with the angle $\phi=0^{\circ}$ and ends with $\phi=0^{\circ}$, thus the duration of each cycle is defined as the time between these two phaseangle values. Figure 10b) presents the histogram of the period durations for one acquisition. It is shown that this duration is mainly close to the expected average $T_{\text {moy }} . U / D=7$, except for some rare episodes occurred when the duration of the cycle was remarkably long $(T . U / D=14)$ or short. This shows that the duration of the vortex shedding cycle can be strongly modulated by the random flow components.

After the phase-identification of each realization, the ensemble of the acquired fields is sorted according to their belonging to one of 40 classes of width $9^{\circ}$. The number of fields in each class is slightly fluctuating about 300 snapshots. The phase-averaged sequence is shown in Figure 14 and will be discussed later in this section.

\subsubsection{Quasi-steady separation of the shear layers from the upstream cylinder}

It was previously mentioned that in FPII the shear layers separate from the upstream cylinder without significant roll-up in the near wake, because large-scale quasi-alternating vortices are observed beyond $x / D=-2$. It was observed in addition that the temporal fluctuations of velocity are weak in this region in comparison with FPI. These facts suggest that the separation 

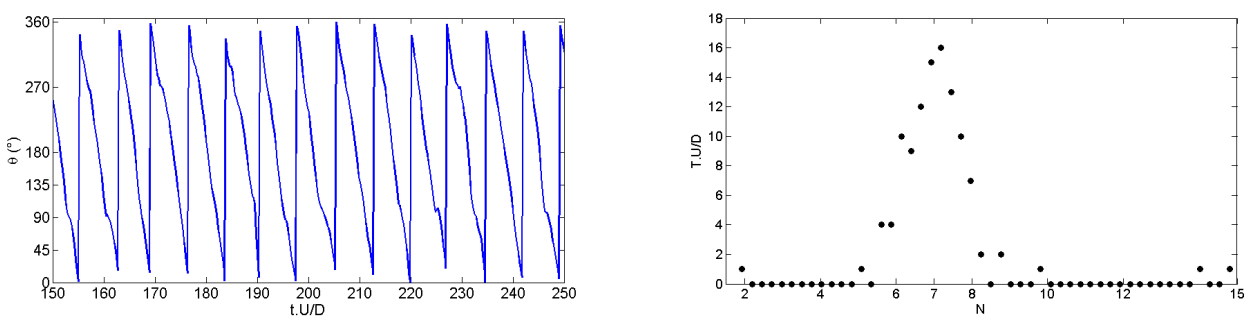

a)

b)

Figure 10: a) Example of a phase angle time series derived from the first two modes POD; b) Time series of each vortex shedding period in the wake of the downstream cylinder. Generally, the duration of the period slightly fluctuates around its averaged value.

from the upstream cylinder in the reattachment pattern is quasi-steady or weakly fluctuating in comparison with the same flow in the co-shedding pattern, thus highlighting a fundamental difference between the two patterns of the bistable regime.

The quasi-steadiness of FPII in the very near region is also confirmed by the spectra of the vertical velocity component at various $\mathrm{X}$-coordinates on the line $y / D=0.68$ (Figure 11). It is shown that up to $x / D=-2$, these spectra display a predominant Strouhal number peak which is progressively attenuated towards near-region positions. This suggests that the flow is dominated by random components in this region. On the other hand, beyond this $X$ coordinate, closer to the downstream cylinder, the spectra display a well distinct fundamental frequency peak at Strouhal number $f . D / U=0.14$. This shows the existence of a significant quasi-periodic motion in the vicinity of the downstream cylinder. The nature of this motion will be discussed in the next sub-section.

In order to analyze the quasi-steady separation by a higher frequency resolution, PIV acquisitions have been carried out at a higher sampling frequency of $8 \mathrm{kHz}$ and using a larger spatial resolution by focusing on a reduced size PIV field in the vicinity of the separation area and along the lower shear layer (Figure 12). This layer is clearly visible from the raw images where it appears like a dark rectilinear streak that breaks downstream to form what seems to be a layer with Kelvin-Helmholtz vortices as a result of the shear layer instability.

An important result of these visualizations is that the position of this 


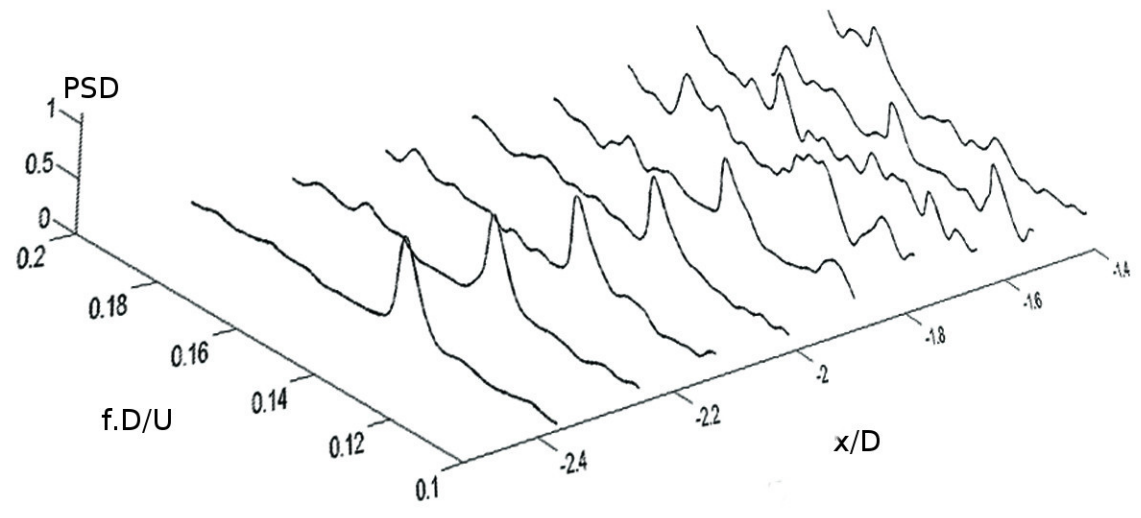

Figure 11: V-velocity spectra at different $\mathrm{X} / \mathrm{D}$ positions on the line $\mathrm{Y} / \mathrm{D}=0.78$ in the wake of the upstream cylinder. Each spectrum is normalised by the maximum PSD.
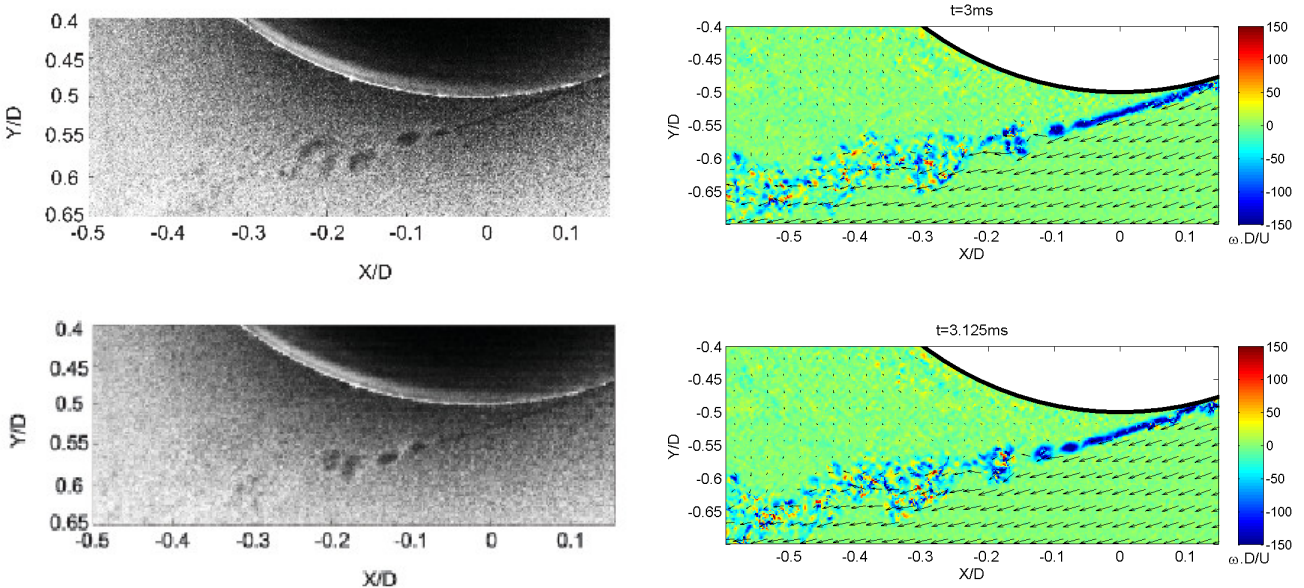

Figure 12: Raw images in the vicinity of the separation point on the upstream cylinder and fields of velocity (vectors) and vorticity (background colour map). 
separated shear layer fluctuates weakly with time, contrarily to FPI where similar visualizations have shown that the layers flap at the vortex shedding frequency. Indeed, the shear layer in FPII seems to keep fairly the same position and thus the same slope during the acquisition. The separation point is out of the view field but can be easily localized as the point where the separation line is tangent to the section of the cylinder. Thus the angular position of this point has been estimated at $\theta=72^{\circ}$ which is close to the position $\theta=75^{\circ}$ estimated by Alam et al (2003) for a single cylinder.

The preceding remarks are confirmed by visualizing the instantaneous velocity and vorticity fields resulting from the cross-correlation analysis of the raw images shown in Figure 12. It is shown that the shear layer clearly delimits the border between the free stream and the stagnation zone. The vorticity is concentrated in the shear layer where it reaches a dimensionless value of $150 D / U$ and seems to be relatively uniform up to the breakdown structure, displaying small vorticity blobs at the location of the first KelvinHelmholtz vortices. Upstream of this breakdown the shear layer is parallel to the free-stream velocity vectors, thus making an angle of $18^{\circ}$ with the $\mathrm{X}$ axis.

By proceeding to a Lagrangian tracking of the Kelvin Helmholtz vortices as well as a spectral analysis of their vertical velocity, we have proven that the predominant frequency peak of $1800 \mathrm{~Hz}$ shown in the spectra of Figure 13 corresponds indeed to the shedding frequency of these vortices, with a Strouhal number of 7 , given that the upstream velocity in this case was $20.6 \mathrm{~m} / \mathrm{s}$ and the cylinder's diameter of $8 \mathrm{~cm}$.

\subsubsection{Alternate reattachment of the shear layers on the downstream cylinder}

In the present section, the focus is made on the behavior of the shear layers as they approach the downstream cylinder. While it is well known that below the critical pitch ratio these layers do reattach on this cylinder, the reattachment dynamics and the subsequent flow structure show a complex phenomenology that has been discussed by several authors. Alam et al (2003) distinguishes between a symmetric reattachment where the two shear layers reattach simultaneously on the cylinder and an alternate reattachment where only one layer reattaches at the same time, the other reattaching a half-period afterwards. In both cases, the reattached layer impacts on the cylinder at the "reattachment point", then separates into "forward" and "backward" shear layers. The first rolls up and forms a large scale vortex upstream, while the second layer adheres to the cylinder then separates near its base. 


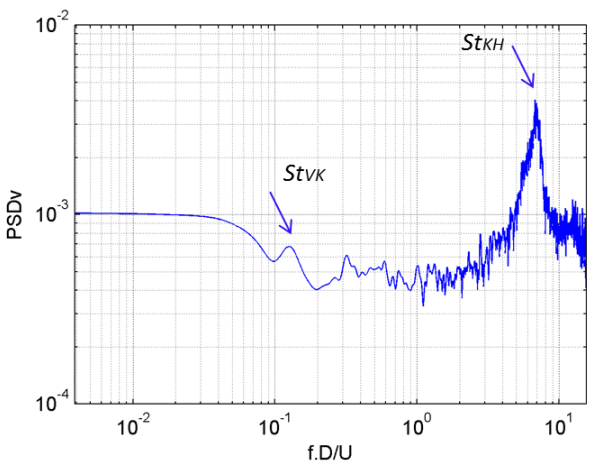

a) $y / D=-0.515$

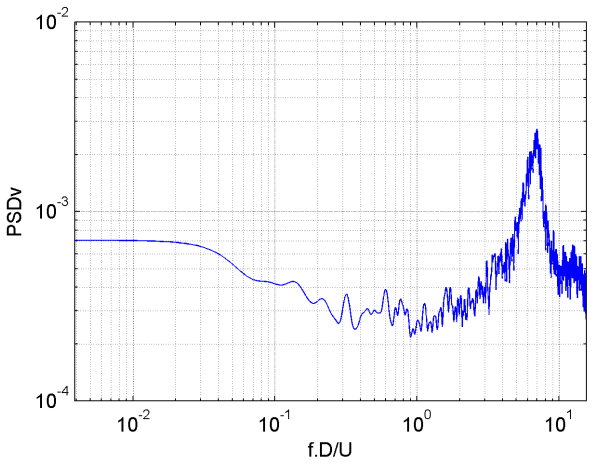

c) $y / D=-0.555$

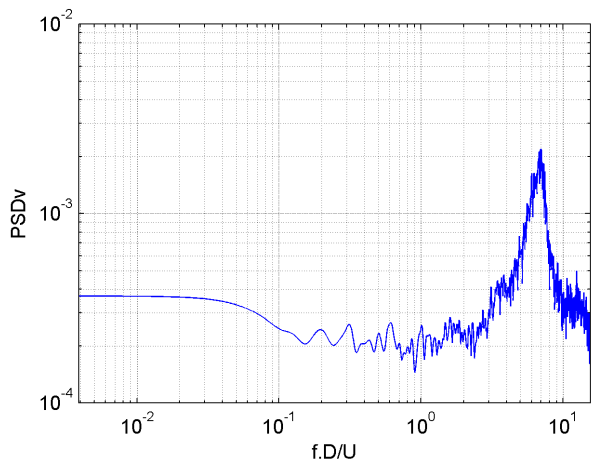

e) $y / D=-0.575$

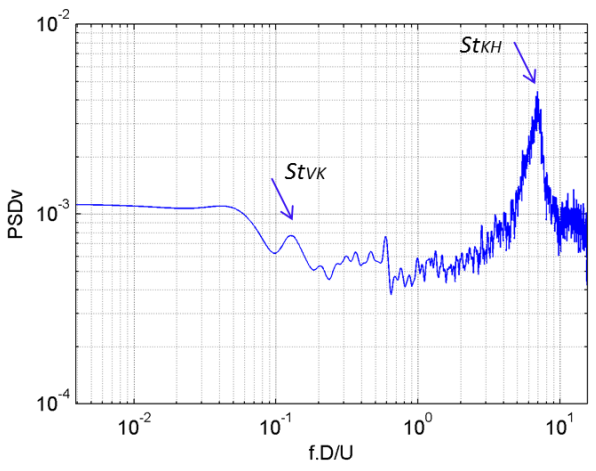

b) $y / D=-0.525$

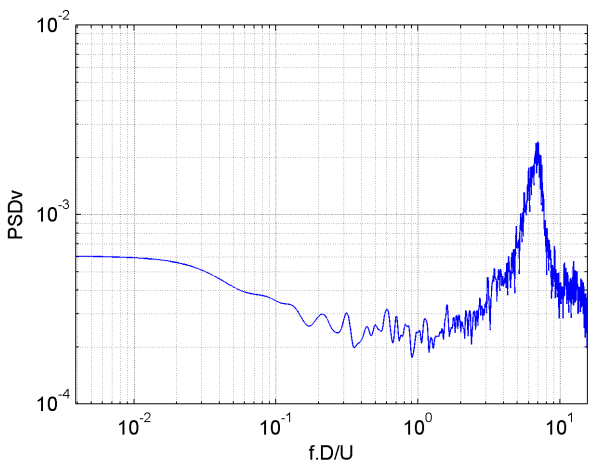

d) $y / D=-0.565$

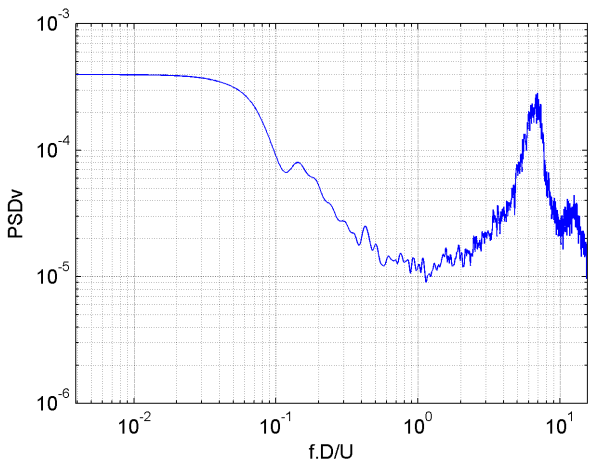

f) $y / D=-0.585$

Figure 13: Power-spectral density of the vertical velocity component at $x / D=-0.17$ and various $y / D$ positions. 

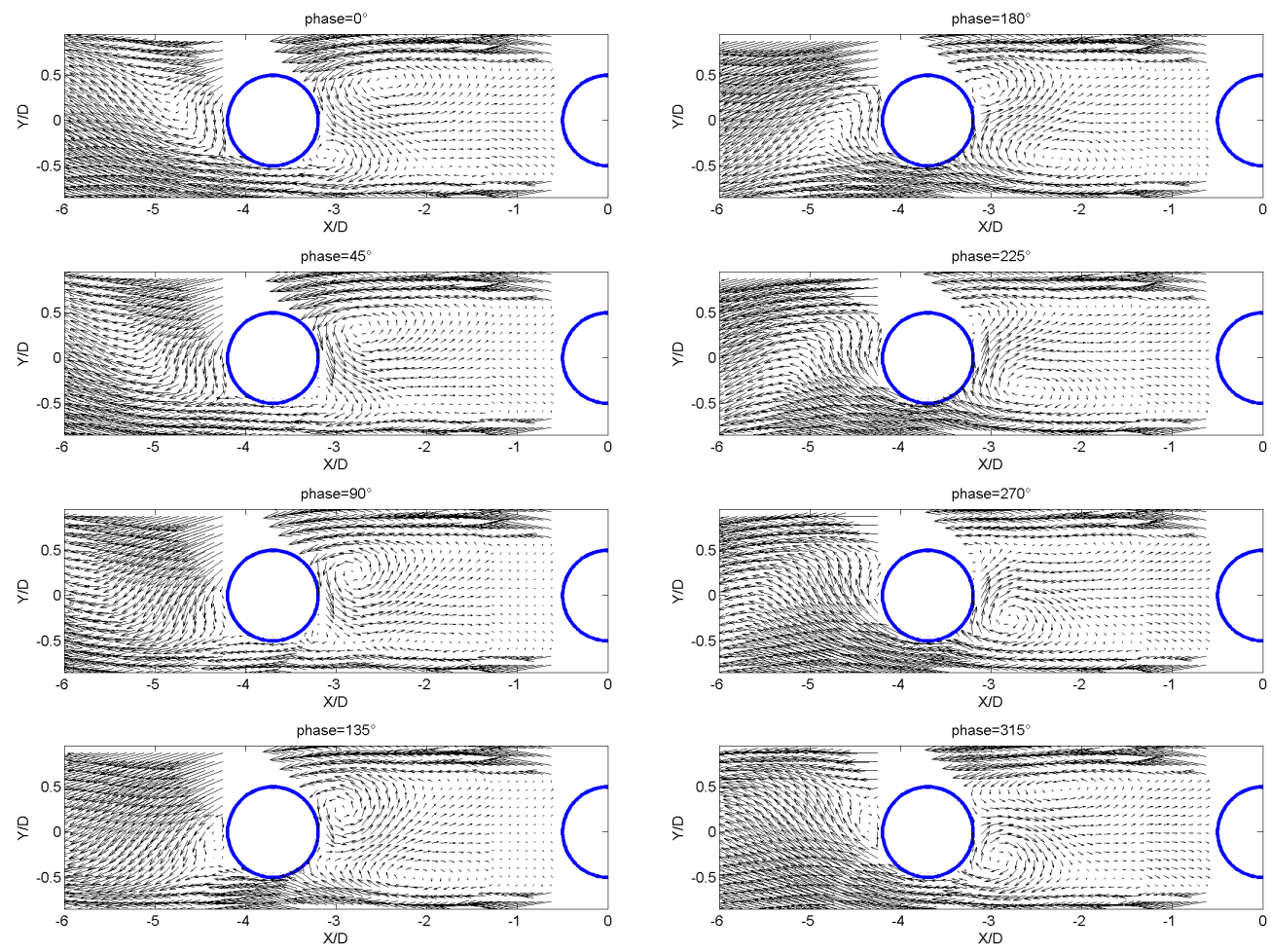

Figure 14: Phase-averaged velocity fields sequence around the downstream cylinder at phase angles $\theta=0^{\circ}, 45^{\circ}, 90^{\circ}, 135^{\circ}, 180^{\circ}, 225^{\circ}, 270^{\circ}, 315^{\circ}$.

In Alam et al (2003) the alternate reattachment is observed for the range of pitch ratios $L / D<3$, while the symmetric reattachment is observed for pitch ratios between $L / D=3$ and the critical pitch ratio. Similar remarks are made by Lin et al. (2002) who report the existence of what is called "recirculation eddies" in the gap between the two cylinders, which can be distributed in a symmetric or asymmetric way according to the pitch ratio. Igarashi (1981) discussed the existence of a "synchronized flow mode" for the pitch range $L / D<2$ where the reattachment is alternate and synchronized with the vortex shedding in the wake of the downstream cylinder.

In the present study, the phase-averaged velocity and vorticity fields have been analyzed to determine the dynamics as well as the reattachment structure, in relation with the flow dynamics past the downstream cylinder; these fields are shown in Figures 14 and 15 respectively. Figure 14 shows a mean ve- 
locity field sequence at phase angles $\theta=0^{\circ}, 45^{\circ}, 90^{\circ}, 135^{\circ}, 180^{\circ}, 225^{\circ}, 270^{\circ}, 315^{\circ}$. Figure 15 shows the phase-averaged fields of the vorticity component perpendicular to the measurement plane. The interaction between the phaseaveraged velocity and phase-averaged vorticity can be modeled by the vorticity transport equation. The present measurements allow further comparisons with numerical studies of this flow. Indeed, turbulence modeling closures often use the phase-averaged decomposition instead of the Reynolds averaged one, in order to capture the coherent structures development representing the resolved turbulence and use adequate turbulence modeling closures for the fluctuating part beyond this averaging, (the modeled turbulence), as for example in the Organised Eddy Simulation (OES) method applied in a cylinder array configuration by Shinde et al (2014) among other. The present experimental study is devoted to the analysis of the phase-averaged fields only, the fluctuations about this average and the related phase-averaged turbulence stresses will be the subject of a forthcoming study.

A first glance at the phase-averaged velocity and vorticity sequences suggests that the two shear layers issued from the upstream cylinder impinge alternatively on the downstream one. Hence the upper shear layer is reattached at the phase angle $\phi=45^{\circ}$ while the lower shear layer seems to stay horizontal even in the wake of the downstream cylinder. The upper shear layer stays reattached up to phase angle $\phi=180^{\circ}$, where the lower one begins to curve towards the downstream cylinder. This shear layer seems to flap at the reattachment frequency but does not present any large scale vortex, as previously mentioned. Moreover, the phase-averaged vorticity seems to gush quasi-steadily from the upstream cylinder towards the downstream one and to display an alternate pattern in the very vicinity of the reattachment zone, then to be alternatively shed in the wake of the downstream cylinder.

The topology and flow dynamics in the near wake of the upstream cylinder seem to be consistent with the quasi-steady behavior previously discussed. Indeed, the averaged velocity distribution around the upstream cylinder is quasi-symmetric up to a distance in the $x / D$ interval $(-1.5,-2)$ and displays an alternate pattern more downstream, yielding slight variations within the period. The sequence clearly shows that the upper and lower horizontal shear layers delimit a broad stagnation zone. These layers concentrate high vorticity values, while the stagnation areas are characterized from a nearly zero vorticity level.

Close to the downstream cylinder, the reattached layers curve towards this cylinder then impact it at the reattachment point. Following the im- 

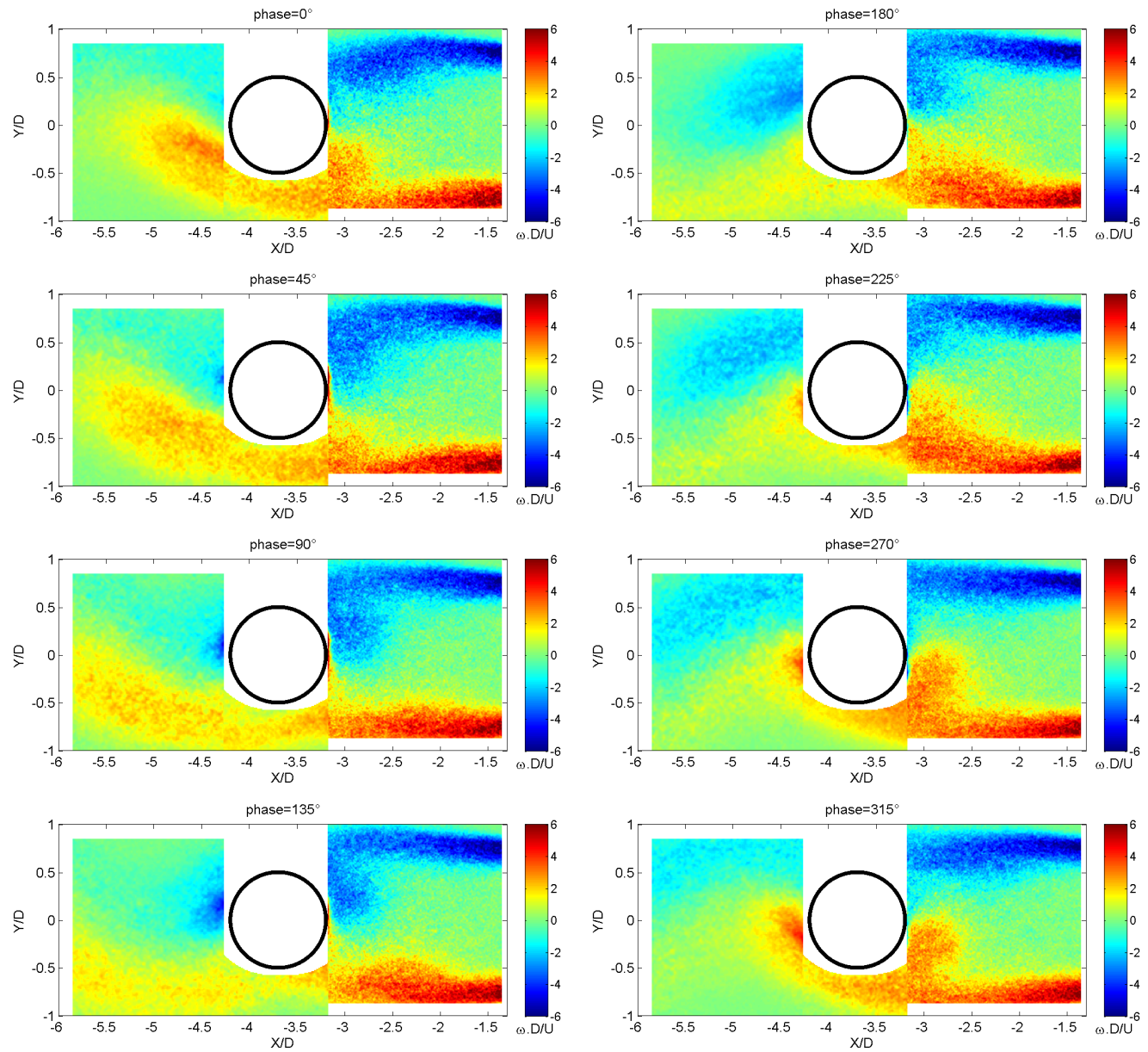

Figure 15: Phase-averaged vorticity fields around the downstream cylinder at phase angles $\theta=0^{\circ}, 45^{\circ}, 90^{\circ}, 135^{\circ}, 180^{\circ}, 225^{\circ}, 270^{\circ}, 315^{\circ}$. 
pact, a bifurcation of the flow occurs around this point and formation of two layers towards the upstream and the downstream cylinders as observed by Alam et al (2003); these are called "backward" and "forward" shear layers respectively. The backward layer rolls up towards the upstream cylinder and forms large vortices of a size close to $D / 2$, which are similar to the "recirculation eddies" previously observed by Lin et al. (2002). These eddies induce an important vertical (upstream or downstream) flow with a velocity close to $U / 2$. Further downstream, these vortices induce a horizontal weak flow towards the upstream cylinder, similar to the "jet like pattern flow" also observed by Lin et al. (2002). In addition, the vorticity fields show that each of the two layers convects the vorticity generated by the upstream cylinder towards the vicinity of the downstream cylinder. Following the bifurcation, the backward shear layer has advected the vorticity towards the upstream cylinder, resulting in a blob of high negative vorticity values between the phase angles $\theta=90^{\circ}$ and $\theta=180^{\circ}$ and positive values between $\theta=225^{\circ}$ and $\theta=315^{\circ}$. As expected, these vorticity blobs accompany the appearance of the recirculation eddies.

The upstream shear layer seems to adhere to the wall of the downstream cylinder then to separate at a position close to the cylinder's base. Further downstream, this shear layer seems to join the non-reattached layer which is not deviated and still remains horizontal. For example, the upper shear layer joins the lower one between $\phi=45^{\circ}$ and $\phi=180^{\circ}$. The vorticity blob visible in the back of the downstream cylinder results from the vorticity advection of the forward layer (see at $\theta=90^{\circ}$ for the upper shear layer and at $\theta=270^{\circ}$ for the lower shear layer). Each of these blobs seems to separate farther from the downstream cylinder, for example at $\theta=135^{\circ}$ for the lower blob, and to be advected downstream. Thus an alternate vorticity detachment is observed in the wake of the downstream cylinder, as in the case of a single cylinder. This vorticity detachment is related to the vortex shedding previously revealed by the first two POD modes. It is finally noted that the vorticity values decrease while moving downstream: at the phase angle $\theta=45^{\circ}$, the vorticity maximum in the lower shear layer exceeds $6 D / U$ upstream of $X / D=-2.5$, whereas this value is lower than $3 D / U$ beyond $X / D=-4.5$. This phaseaveraged vorticity reduction of more than half is mainly due to the viscous dissipation.

In conclusion, the phase-averaged velocity fields show that the shear layers issued from the upstream cylinder reattach alternatively on the downstream cylinder. Therefore the quasi-periodic motion previously discussed 
matches with this alternate reattachment. Moreover the averaged vorticity sequence suggests that the reattachment is accompanied by the formation of a high vorticity blob in the rear part of the downstream cylinder that separates and is advected downstream. Therefore, the vortex shedding past the downstream cylinder in the present study is synchronous with the quasiperiodic reattachment of the shear layers on this cylinder, in agreement with the "synchronized flow mode" observed by Igarashi (1981), .

\subsubsection{Discussion}

The results presented in the previous section allowed highlighting a major difference between the topologies and the dynamics of the flow around each cylinder in the reattachment regime (FPII). While the flow around the upstream cylinder is relatively weakly fluctuating and quasi-symmetrically distributed in the near region, the flow around the downstream cylinder is quasi-periodic and displays alternating reattachment of the two shear layers upstream of this cylinder. These elements can be summarized in the simplified scheme of the reattachment flow presented in Figure 16.

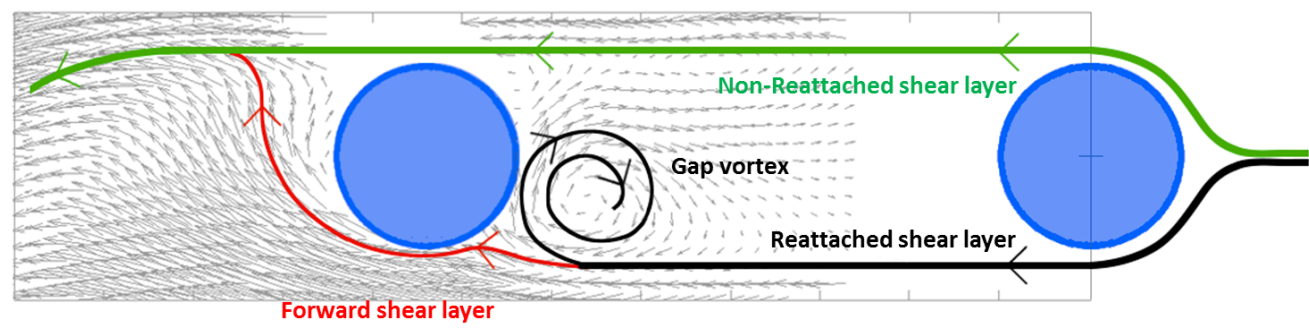

Figure 16: Schematic diagram of the alternate reattachment

Moreover, it is interesting to relate these observations to other studies concerning differences in aerodynamic loads and surface pressure distributions between the two cylinders. For pitch values right smaller than the critical one, it is reported that the pressure and forces fluctuations are more important for the downstream cylinder than for the upstream one. Alam et al (2003) show that for the pitch ratio $L / D=3.5$, the fluctuating surface pressure coefficient is low $\left(C_{p f}=0.025\right)$ and is fairly invariant with the angular position. For the downstream cylinder, this coefficient varies considerably 
as a function of the angular position between $C_{p f}=0.15$ and $C_{p f}=0.35$, these values being ten times larger than those for the upstream cylinder. The lift fluctuations present the same tendency: Alam et al (2003) show that the fluctuating base pressure is close to $C_{L f}=0.05$ for the upstream cylinder and up to $C_{L f}=0.4$ for the downstream cylinder. Moreover, this study notes the absence of a Strouhal peak on the lift spectrum for the upstream cylinder, while it is visible for the downstream cylinder.

Therefore, the present study is in agreement with these observations on the fact that the flow in the immediate vicinity of the upstream cylinder is quasi-steady and does not present a sufficiently significant quasi-periodic coherent component, to induce a quasi-periodic lift on this cylinder. Furthermore, the alternate reattachment imposes important fluctuations of the surface pressure, as reported in the mentioned studies. These fluctuations are the highest at the reattachment point. The asymmetry of the velocity field shown by the present TRPIV measurements induces an instantaneous momentum flux through the cylinder surface, which can be related to the important lift and drag fluctuations. It would be interesting to identify in a future study the cycle phases corresponding for example to the maxima and minima of the aerodynamics forces.

\section{Conclusions}

The present study investigated the flow around two critically spaced circular cylinders in cross flow at a high sub-critical Reynolds number, by means of time resolved PIV. The pitch ratio between the two cylinders, $L / D=3.7$ was in the critical range for the transition between the reattachment and the co-shedding regimes. The flow observed was indeed bistable. The TRPIV measurements quantified the flow structure in case of the alternating flow pattern FPI and the reattached one, FPII, in terms of averaged velocity and Reynolds stress fields. The Reynolds stresses have been found considerably weaker in case of FPII. The corresponding shedding frequencies have been quantified in terms of Strouhal numbers for FPI and FPII. A drastic decrease of the Strouhal number in case of FP2 has been noted. The shear layers past the separation points of the upstream cylinder are governed by a KelvinHelmholtz instability associated with smaller-scale vortices, that the present study was able to capture and quantify their shedding frequency thanks to a high-speed camera providing a sampling rate of $8 \mathrm{kHz}$. The reattachment flow pattern (FPII) has been analyzed in detail concerning the shear layer 
and vortex dynamics past the upstream cylinder, the reattachment nature near the impact on the downstream cylinder, the gap flow and the wake structure past the downstream cylinder. Particular attention has been paid to the organized motion of the flow. To this aim, a POD analysis has been carried out, allowing phase-averaged decomposition of the flow in respect of the quasi-periodic motion, as well as a modal analysis concerning the most energetic modes governing the present system. It has been shown that the first decade of POD modes contain a substantial part of the whole energy and that these modes are governed by pronounced frequency peaks. The two first modes have a major contribution to the kinetic energy and have been used for the phase-average analysis. Thanks to this, the overall PIV measuring planes have been associated one another and a detailed discussion of the phase-averaged flow structure has been possible. These novel elements in the state of the art allowed drawing main differences of the reattachment region comparing with the near region past the upstream cylinder, the gap flow and the region past the downstream cylinder. The synchronization mechanism between the reattachment, the gap flow and the vortex shedding in the wake of the downstream cylinder has been shown. The present study has highlighted the coexistence of a quasi-steady flow near the upstream cylinder and a quasi-periodic flow in the gap region between the two cylinders. It has been shown that the alternate reattachment defines both the structure and the dynamics of the gap flow. Therefore the gap flow near the downstream cylinder consists of the alternate appearance of upper and lower gap vortices at the same frequency as the reattachment. It has been shown that the vortex shedding in the wake of the downstream cylinder is synchronized with the reattachment.

Thus, the present study contributes among other to a better understanding of the reattachment regime.

\section{Acknowledgments}

This study has been accomplished in the context of the French research program of the "Agence Nationale de Recherche" - ANR BARESAFE, "Simulation of Safety Bareer reliability", coordinated by EDF and under the partnership of EDF, CEA, AREVA, "Institut de Recherche en Informatique de Toulouse" - IRIT, "Institut de Mécanique des Fluides de Toulouse" - IMFT, "Laboratoire des sciences de l'ingénieur, de l'informatique et de l'imagerie", Université de Strasbourg - ICUBE. Part of this study was carried out in 
the national research platform RTRA-SMARTWING, www.smartwing.org, funded by the French Foundation STAE, "Sciences et Technologies pour l'Aéronautique et l'Espace". The authors are grateful to Mrs Muriel SabaterBoutic, reprographics service of IMFT, for her contribution in the figures.

\section{References}

Adrian, R. J., Westerweel, J., 2010. Particle Image Velocimetry. Cambridge University Press

Alam, M. M., Moriya, M., Takai, K., Sakamoto, H., 2003. Fluctuating fluid forces acting on two circular cylinders in a tandem arrangement at a subcritical Reynolds number. J. of Wind Engineering an Industrial Aerodynamics. Vol. 91(1-2), 139-154.

Alam, M. M., Meyer, J. P., 2011. Two interacting cylinders in cross flow. Physical Review E. Vol. 84(5), 056304.

Auteri, F., Belan, M., Gibertini, G., Grassi, D., 2008. Normal flat plates in tandem: An experimental investigation. J. of Wind Eng. and Ind. Aerodynamics. Vol. 96(6-7), 872-879.

Berkooz, G., Holmes, P., Lumley, J. L., 1993. The proper orthogonal decomposition in the analysis of turbulent flows. Annual Review of Fluid Mechanics. Vol. 25, 539-575.

Braza, M., Perrin, R., Hoarau, Y., 2006. Turbulence properties in the cylinder wake at high reynolds number. J. of Fluid and Struct. 22, 7551-771.

Cantwell, B. J., Coles, D., 1983. An experimental study of entrainment and transport in the turbulent near wake of a circular cylinder. J . of Fluid Mechanics. Vol. 136, 321-374.

Connors, H. J., 1970. Flow induced vibration of heat exchanges. New York:ASME Fluidelastic vibrations of tube arrays excited by cross flow, $42-56$.

Ferreira-Perez, S., 2013. Simulation numérique et modélisation de l'interaction fluide-structure et étude vibratoire de deux cylindres en tandem nombre de Reynolds élevé. Engineering diploma thesis, ENSEEIHTIMFT. 
Fu, S., Haase, W., Peng, S. H., Schwamborn, D., 2012. Progress in Hybrid RANS-LES Modelling. Notes on Numerical Fluid Mechanics and Multidisciplinary Design 117, 59-75.

Granger, S., Paidoussis, M., 1996. An improvement to the quasi-steady model with application to cross-flow-induced vibration of tube arrays. J. Fluid Mech. (320), 163-184.

Hussain, A. K. M. F., Reynolds, W. C., 1970. The mechanics of an organized wave in turbulent shear flow. J . of Fluid Mechanics. Vol. 41, part 2, 241258.

Igarashi, T. 1981. Characteristics of the Flow Around Two Circular Cylinders Arranged in Tandem. I. JSME Bulletin. Vol. 24, Feb. 1981, 323-331.

Iwaki, C., Cheong, K. H., Monji, H., Matsui, G., 2004. PIV Measurement of the Vertical Cross-flow Structure over Tube Bundles. Experiments in Fluids. Vol. 37(3), 350-363.

Jenkins, L., Khorrami, M., Choudhari, M., McGinley, C., 2005. Characterization of Unsteady Flow Structures Around Tandem Cylinders for Component Interaction Studies in Airframe Noise. AIAA paper 2005-2812.

Khalifa, A., Weaver, D. S., Ziada, S., 2013. Modeling of the phase lag causing fluidelastic instability in a parallel triangular tube array. J. of Fluids and Structures. Vol. 43, 371-384.

Kitagawa, T., Ohta, H., 2008. Numerical investigation on flow around circular cylinders in tandem arrangement at a subcritical Reynolds number. J. of Fluids and Structures. Vol. 24(5), 680-699.

Kim, M. K., Kim, D. K., Yoon, S. H., Lee, D. H., 2008. Measurements of the flow fields around two square cylinders in a tandem arrangement. J. of Mechanical Science and Technology. Vol. 22(2), 397-407.

Kourta, A., Boisson, H. C., Chassaing, P., Minh, H. Ha, 1987. Nonlinear interaction and the transition to turbulence in the wake of a circular cylinder. J. of Fluid Mechanics. Vol. 181, 141-161.

Keogh, D., Meskell, C., 2014. Bi-stable flow in parallel triangular tube arrays with a pitch-to-diameter ratio of 1.375. Proceedings of the ASME 
2014 Pressure Vessels \& Piping Conference, PVP2014, July 20-24, 2014, Anaheim, California, USA.

Lin, J.-C., Yang, Y., Rockwell, D., 2002. Flow past two cylinders in tandem: instantaneous and averaged flow structure. J. of Fluids and Structures. Vol. 16(8), 1059-1071.

Lockard, D., Khorrami, M., Choudhari, M., Hutcheson, F., Brooks, T., Stead, D., 2007. Tandem Cylinder Noise Predictions. AIAA Paper 20073450 .

Mahir, N., Rockwell, D., 1996. Vortex formation from a forced system of two cylinders. Part II: side-by-side arrangement. J. of Fluids and Structures. Vol. 10(5), 491-500.

Mahon, J., Meskell, C., 2012. Surface pressure survey in a parallel triangular tube array. J. of Fluids and Structures. Vol. 34, 123-137.

Marple, S. L., 1987. Digital Spectral Analysis with Applications. PrenticeHall Series in Signal Processing. Prentice Hall publisher.

Neuhart, D., Jenkins, L., Choudhari, M., Khorrami, M., 2009. Measurements of the Flowfield Interaction Between Tandem Cylinders. AIAA Paper 20093275 .

Olinto, C. R., Indrusiak, M. L. S., Mller, S. V., 2006. Experimental Study of the Bistable Flow in Tube Arrays. J. of the Brazilian Soc. of Mech. Sci. \& Eng. Vol. 8(2), 233-241.

Olinto, C. R., Indrusiak, M. L. S., Endres, L. A. M., Mller, S. V., 2009. Experimental study of the characteristics of the flow in the first rows of tube banks. Nuclear Engineering Design. Vol. 239, 2022-2034.

Paidoussis, M. P., Price, S. J., 1988. The mechanisms underlying flow-induced instabilities of cylinder arrays in crossflow. J. of Fluid Mech. 187, 45-59.

Paul, S. S., M.F. Tachie, Ormiston., S. J., 2007. Experimental study of turbulent cross-flow in a staggered tube bundle using particle image velocimetry. Int. J. of Heat and Fluid Flow. Vol. 28(3), 441-453. 
Perrin, R., Braza, M., Cid, E., Cazin, S., Barthet, A., Sevrain, A., Mockett, C., Thiele, F., 2007. Obtaining phase averaged turbulence properties in the near wake of a circular cylinder at high Reynolds number using POD. Experiments in Fluids. Vol. 43(2-3), 341-355.

Sirovich, L. , 1987. Turbulence and the dynamics of coherent structures. Quarterly of Applied Mathematics. Vol. 5, 561-590.

Shinde, V., Marcel, T., Hoarau, Y., Deloze, T., Harran, G., Baj, F., Cardolaccia, J., Magnaud, J.P., Longatte, E., Braza, M., 2014. Numerical simulation of the fluid-structure interaction in a tube array under cross flow at moderate and high Reynolds number. J. of Fluids and Structures. Vol. 47, 99-107

Sumner, D., Wong, S. S. T., Price, S. J., Paidoussis, M. P., 2000. Fluid behaviour of side-by-side circular cylinders in steady cross-flow. J. of Fluids and Structures. Vol. 13, 309-338.

Sumner, D., Price, S. J., Paidoussis, M. P., 2000. Flow-pattern identification for two staggered circular cylinders in cross-flow. J. of Fluid Mechanics. Vol. 411, 253-303.

Sumner, D., 2010. Two circular cylinders in cross-flow: A review. J. of Fluids and Structures. Vol. 26(6), 849-899.

Van Oudheusden, B. W., Scarano, F., Hinsberg, N. P., Van Watt, D. W., 2005. Phase-resolved characterization of vortex shedding in the near wake of a square-section cylinder at incidence. Experiments in Fluids. Vol. 39(1), 86-98.

Xu, G., Zhou, Y., 2004. Strouhal Numbers in the Wake of Two Inline Cylinders. Experiments in Fluids. Vol. 37(2), 248-256.

Zdravkovich, M. M., 1991. Flow Around Circular Cylinders Volume 1: Fundamentals. Oxford University Press USA.

Zhou, Y., Yiu, M. W., 2006. Flow structure, momentum and heat transport in a two-tandem-cylinder wake. J. of Fluid Mechanics. Vol. 548, 17-48. 